\title{
Somatic genetics of CDR3 control TCR V- domain rotational probability and germline CDR2 scanning of polymorphic MHC
}

Joseph Murray ( $\sim$ jsmurray@xenolausgenetics.com )

Xenolaus Genetics LLC

\section{Research Article}

Keywords: T-cell receptor (TCR), Major Histocompatibility Complex (MHC), Clonal Selection, MHC Restriction, Protein Chemistry, Mathematical Biology

Posted Date: March 15th, 2021

DOl: https://doi.org/10.21203/rs.3.rs-253324/v1

License: (c) (1) This work is licensed under a Creative Commons Attribution 4.0 International License.

Read Full License 
1 Title Page

2

3

4

5

6 Full title:

7 Somatic genetics of CDR3 control TCR V-domain rotational probability

8 and germline CDR2 scanning of polymorphic MHC

10 Running title:

11 Genetic calculus of TCR binding class-II pMHC

12

13

14

15

16

17

18

19

20

21

$22+$ Xenolaüs Genetics LLC

23 Los Angeles, CA, 90024, USA

24 


\title{
Somatic genetics of CDR3 control TCR V-domain rotational probability and germline CDR2 scanning of polymorphic MHC
}

\author{
Joseph S. Murray ${ }^{+*}$ \\ †Xenolaüs Genetics LLC \\ Los Angeles, CA UNITED STATES \\ *Email Author: Dr. J. S. Murray, Ph.D. (jsmurray@xenolausgenetics.com)
}

\section{Abstract:}

The mechanism which adapts the T-cell antigen receptor (TCR) within a given major histocompatibility complex (MHC/HLA) genotype is essential for protection against pathogens. Historically attributed to relative affinity, genetically vast TCRs are surprisingly focused towards a micromolar affinity for their respective peptide (p) plus MHC (pMHC) ligands. Thus, the somatic diversity of the TCR with respect to MHC-restriction, and (ultimately) to pathogens, remains enigmatic. Here, we derive a triple integral solution (from fixed geometry) for any given $V$ domain in TCR bound to PMHC. Solved complexes involving HLA-DR and HLA-DQ, where genetic linkage to the TCR is most profound, were examined in detail. Certain $\mathrm{V}$ domains displayed rare geometry within this panel-specifying a restricted rotational probability/volumetric density (dV). Remarkably, hydrogen $(H)$ bond charge-relays distinguished these structures from the others; suggesting that CDR3 binding chemistry dictates CDR2 contacts on the opposite MHC-II alpha helix. Together, these data suggest that TCR recapitulate $d V$ and specialise target pMHC recognition, i.e., a dynamics alternative to a relative TCR-affinity based mechanism.

\section{Keywords:}

T-cell receptor (TCR); Major Histocompatibility Complex (MHC); Clonal Selection; MHC Restriction; Protein Chemistry; Mathematical Biology

\section{Introduction:}

T-cell antigen receptors (TCR) and antibodies (slg) are individualized within each precursor of a given T-cell or B-cell clone. TCR ( $\alpha / \beta$ type) have genetically variable $(V)$ domains, wherein complementary determining regions (CDR1-CDR3) contain closest amino acid (a.a.) contacts with the peptide $(\mathrm{p})$ plus major histocompatibility complex (MHC) protein (together abbrev., pMHC) composite ligand. CDR1 and CDR2 are encoded in the germline, via the particular Vregion DNA segment involved in the RAG1/RAG2 recombination mechanism responsible for somatic construction, together with the D- and/or J-segment(s), of the third, CDR3 [1-5]. This paper seeks to understand two puzzles of the TCR-pMHC interaction, wherein a novel 
examination of the first can be used to re-examine the second in the context of existing evidence. Firstly, compared to antibodies and indeed other protein:protein binding reactions, TCRs display quite low ( $\mu \mathrm{M})$ binding affinities for pMHC ligands [6-8]. Nevertheless, like antibodies, TCRs have exquisite specificity, where single peptide or MHC a.a. changes can dictate T-cell reactivity or non-reactivity [9-17]. Second, the nature of T-cell selection in the thymus prior to seeding secondary lymphoid tissues is thought to operate on the basis of this same relative TCR:pMHC affinity, where overt 'self' peptide plus self-MHC recognition (negative selection) leads to deletion from the repertoire, as does a complete lack of recognition (positive selection) [4]. This intermediate TCR:PMHC binding in the fetal thymus forms the basis of 'simultaneous' self plus pathogen recognition in adulthood, i.e., the MHC-restricted adaptive immune response $[5,18]$. Somatic CDR3 loops are roughly positioned atop the peptide component in solved TCR:pMHC complexes [9-18]; although more recent investigations have challenged the notion that the most diverse component of the ligand (the peptide) is particularly interfaced with the most diverse regions of the receptor (CDR3 $\alpha / \beta)[12,19]$.

For example, we previously reported that one of the CDR3 loops (CDR3 $\alpha$ ) also makes consistently close contacts with a conserved HLA-A component, i.e., the R65-motif (a.a., R65-XX-K68-A69-X-S71-Q72) [20]. Burrows et al., also found conserved CDR3 contacts directly with MHC-I [21]. More recently, Sharon et al., formally linked germline TCR usage to HLA, particularly via the class-II alleles [18]. Here, the STCR-Dab (www. opig.stats.ox.ac.uk/webapps/stcrdab/) was used to identify all current TCR complexes involving HLA-DR and HLA-DQ (3.0 ̊ resolution cut-off). Note, also included are PDB 4H1L, at $3.3 \AA$, and $3 T O E$, at $4.0 \AA$ (see below). A simple relationship between the observed (i.e., measured) CDR3-CDR2 "pitch" angle for a given $V$ domain and its predicted (i.e., calculated) CDR3-CDR2 pitch angle was observed [20]. Here, a linear relationship between calculated pitch and a new measure, " $d V$ " - by multivariable calculus, was found. As is discussed, $d V$ interprets V-domain orientation into a rotational probability involving an apparent CDR2-MHC $\alpha$-helix scanning function $(d \theta)$. While $d V$ were unique for each TCR on each pHLA-DR, one TCR displayed a dramatic restriction in $\alpha \mathrm{V}$ for $\mathrm{V} \beta$. This was isolated to a charge-relay $\mathrm{H}$-bonding mechanism for CDR3 $\beta$; hence, the chemistry of "somatic-TCR" dictates positioning of "germline-TCR". Within the seven PHLA-DQ structures, the two highly-restricted dV TCR (PDB 4OZG \& 4OZH) displayed distinct, yet functionally similar mechanisms that shifted the same TCR:MHC H-bond by one $\mathrm{MHC}$ a.a. position (relative to the nominal $d V$ structure, 4OZF). This involves an additional $\mathrm{H}$ bond (MHC:MHC) in suitable charge-relay mechanisms of $4 \mathrm{OZG}$ and $4 \mathrm{OZH}$.

\section{Results:}

PDB files of solved TCR:pHLA-DR and TCR:pHLA-DQ structures were used to investigate $V$ domain geometry amongst the available complexes, i.e., involving similar (but different) TCR, and/or similar (but different) pMHC. All of these structures share the canonical (diagonal) orientation of the TCR over PMHC, which was one of the earliest observed similarities between different complexes [13]. As indicated in the summary tables (Tables 1A-1D; separation into 
different tables is simply for clarity) all sequences for each component of all of these structures are available under the appropriate PDB file name at the $N C B I$ (www.ncbi.nlm.nih.gov).

\section{TCR-pMHC Geometry}

Shown in Figures $1 \mathrm{~A}$ and $1 \mathrm{~B}$ is an example of the geometry analysis based on fixed a.a. positions in the HLA-DR/HLA-DQ grooves (e.g., $P D B$ 1J8H). We originally used Euler angles (www.mathword.wolfram.com/EulerAngles.html) to establish the basic method (for a TCR$\mathrm{V} \alpha$ cohort in pHLA-A2 complexes) [20]; here, the method was modified for the available solved TCR-pMHC-II structures (summarized in Tables 1A-1D). In brief, the analysis is based on measuring three angles corresponding to the twist $(\omega)$, tilt $(\lambda)$, and sway $(\sigma)$ of each $V$ domain over the $\mathrm{pMHC}$.
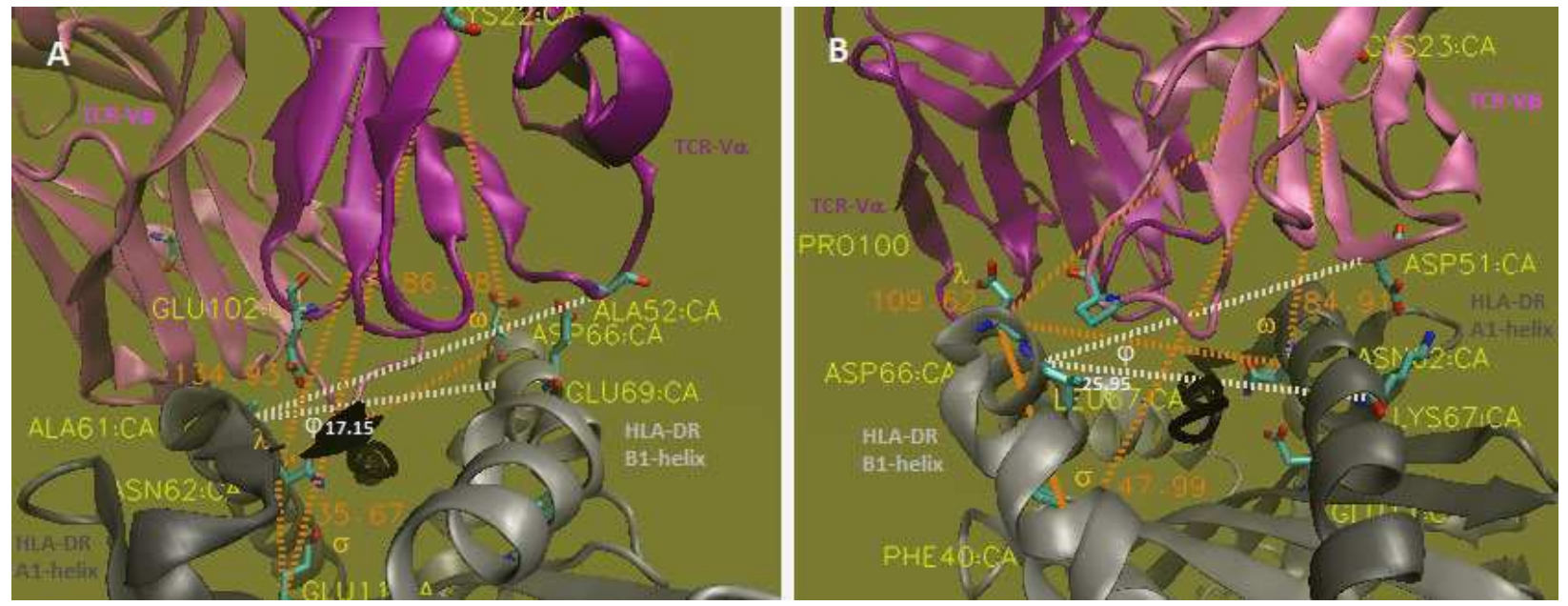

Figure 1 The twist/tilt/sway of TCR-V $\alpha$ and $-\mathrm{V} \beta$ relative to pMHC-II (A \& B, respectively). Illustrated by the example of $1 \mathrm{~J} 8 \mathrm{H}$. This same analysis was performed on all 19 structures (Tables 1A-1D). All angle measurements were from $C_{\alpha}$ in $V M D-1.9 .1$ (www.ks.uiuc.edu) used to examine the diversity through the 38 V-domains: (i) in-plane to the MHC-groove \{twist $=\omega\}$, with (ii) displacements perpendicular to the groove $\{t i l t=\lambda\}$, including (iii) side-to-side variation $\{s w a y=\sigma\}$. The a.a. positions used as coordinates for angular measures (dotted orange vectors) across structures were fixed and are labelled (see text). The measured incline of a $V$ domain, or "pitch" $\left\{\right.$ pitch $\left.=\varphi_{m}\right\}$, is shown by white dotted vectors and could be approximated by the equation: $\varphi_{c}=[\sigma \div(\lambda+\sigma)]_{\omega}$ (see text, Tables 1A-1D). Side chains of a.a. in measurements are shown by CPK licorice; $\mathrm{C}_{\alpha}$-backbones are in new cartoon and labelled; peptides are in black. Note from $A$ to $B$ the view of the structure rotates $180^{\circ}$. Figures are the original output of the $P D B$ file as analysed in VMD-1.9.1. Best viewed at $100 \%$.

For HLA-DR a vector from the DRA a.a. N62 alpha-carbon (N62:C $\alpha$ ) to DRB1/3/5 a.a. D66 (D66:C $\alpha$; $\alpha$ used for measurements unless otherwise noted) bisects the MHC-groove from the DRA $\alpha$-helix to the DRB1/3/5 $\alpha$-helix, then the angle at D66 to the $V \alpha$ central cysteine (C22) is computed with the VMD angle-label tool. This $\omega$-angle can be seen in Figure $1 \mathrm{~A}(\mathrm{~V} \alpha)$ and $1 \mathrm{~B}$ (V $\beta$; where the vectors run D66:N62:C23) as dotted orange lines near the melon-coloured " $\omega$ " symbol, $86.88^{\circ}(\mathrm{V} \alpha), 84.91^{\circ}(\mathrm{V} \beta)$. The E11 (beta-sheet floor) to N62 to C22 (tilt-angle) is similarly shown (1A) near the " $\lambda$ " symbol, $134.93^{\circ}$; for $V \beta$, D66 is used (1B), F40:D66:C23, at 
$109.62^{\circ}$. Finally the $\sigma$-angle is measured from N62:E11:C22 (V $\left.\alpha\right)$ or D66:F40:C23 $(\mathrm{V} \beta)$, here at $35.67^{\circ}$ and $47.99^{\circ}$, respectively. For HLA-DQ TCR:PMHC structures, $V_{\alpha}$ twist is based on a DQA a.a. N62:C $\alpha$ to DQB D66:C $\alpha$ vector bisecting the groove, where the angle vertex is at $D 66$ to $V \alpha$ $\mathrm{C} 23: \mathrm{C} \alpha$. Here, N11:C $\alpha$ (not E11 found in DR) to the N62 vertex to $\mathrm{C} 23$ defines tilt, and the reverse N62:N11:C23 is sway. For the DQ V $\beta^{\prime}$ s, angle a.a. are the same as in DR structures. Specifically, twist is defined by D66:N62:C23, tilt by F40:D66:C23, and sway as D66:F40:C23 (Table 1D). We formulated the equation:

$$
\varphi_{c}=\mathbf{k} \varphi_{m}=[\sigma \div(\lambda+\sigma)] \omega
$$

that predicts the pitch of a given $V$ domain (pitch $=$ angle $\varphi$ ) from the $\omega, \lambda$, and $\sigma$ (orientation). By contrast, measuring the $\varphi$-angle is based on finding the closest contact between the domain CDR2 and the $\alpha$-helix that is opposite CDR3 across-the-groove, N62 (for $V \alpha$ ), or D66 (for V $\beta$ ) (yellow highlighted in Tables 1A-1D). The vectors are directed from that CDR2 a.a. to the across-the-groove $\alpha$-helix a.a. in closest contact with CDR3, then back to the $\alpha$-helix a.a. that is in contact with said CDR2 a.a. (white dotted lines, Figs. 1A \& 1B). In Tables 1A-1D the calculated $\left(\varphi_{c}\right)$ and measured $\left(\varphi_{m}\right)$ pitch angles are shown along with the $k$ values. Thus, to the extent $k$ approaches 1.00 indicates correlation between the measured pitch and calculated pitch values. Statistics: HLA-DR group: mean $k=0.99 \pm 0.36(s), n=24, t=0.915, \mu_{0}=1.00, p=$ 0.376, 95\% Cl, 0.880-1.10; (paired Student's $t$-test; www.graphpad.com/quickcalcs; www.select-statistics.co.uk). HLA-DQ group: mean $k=0.88 \pm 0.35$ (s), $n=14, t=1.24, \mu_{0}=1.00$, $p=0.234,95 \% \mathrm{Cl}, 0.750-1.01$. Overall (DR \& DQ): mean $k=0.95 \pm 0.35(\mathrm{~s}), n=38, t=0.85$, $\mu_{0}=$ $1.00, p=0.402,95 \% \mathrm{Cl}, 0.870-1.03$.

\section{Triple Integrals}

We observed that the closest CDR2 $\alpha$ contacts with the $\alpha 2$-helix 'across-the-groove' from the conserved R65 contact with CDR3 $\alpha$ in HLA-A2 structures specified a polymorphic region from a.a. H/R151 to a.a. A158 [20]. Similarly, the CDR2 $\alpha$ closest contacts range for the DR structures here (Tables 1A-1C) implicate a polymorphic DRB $\alpha$-helix range from a.a. E69 to a.a. T77; for CDR2 $\beta$, a DRA $\alpha$-helix contacts range: a.a. Q57 to a.a. K67. For the DQ complexes: CDR2 $\alpha$ contacts range is the polymorphic DQB $\alpha$-helix a.a. E69 to a.a. D76; and for CDR2 $\beta$, DQA: Q57 to H68, is also polymorphic (Table 1D). Thus, the hypothesis that if these $\alpha$-helical a.a. are indeed swept/scanned by CDR2 of the V-domain, that one should be able to model such with integration from spherical coordinates in each structure [22, 23]. To normalize the equation across structures and use defined geometry, the central cysteine (C22/23) was chosen as a point rotating from the fixed a.a. position that defines twist, tilt, and sway (Figs. 1 \& 2). As shown in Figure 2A \& 2B, the coordinates model a 'slice' of a cone, where for each of the $38 \mathrm{~V}$ domains a measured distance was taken between the $\mathrm{C} 22 / 23$ and said $\alpha$-helix a.a. (e.g., 2IAM $V \beta$ measures $22.12 \AA)$-this is the $r h o(\rho)$ distance in the equation. The other measure was the angle $\phi$, which is simply the difference from $180^{\circ}$ of the previously determined tilt angle $(\lambda)$. The other variables were derived by trigonometry (see Fig. 2B). 


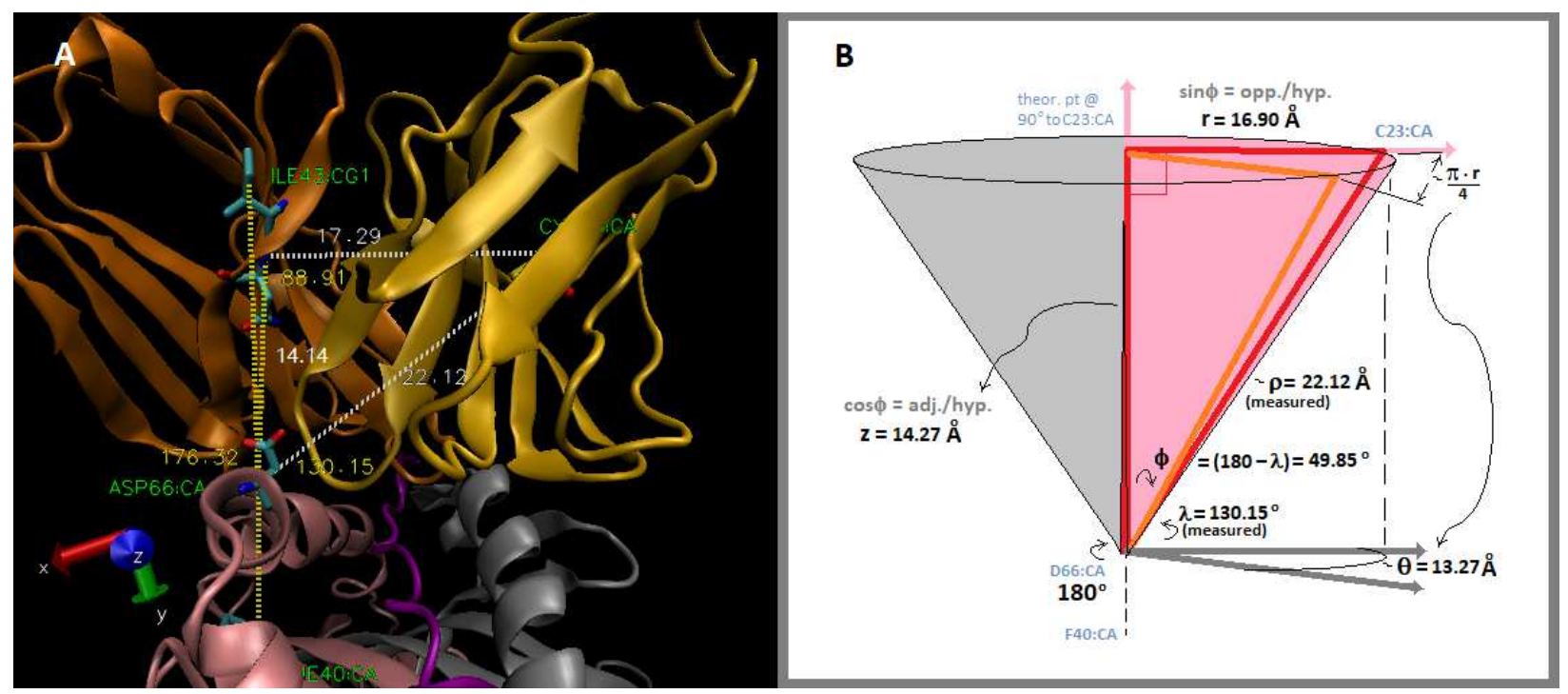

Figure 2 Derivation of the V-domain rotational volumetric-density equation. The same a.a. positions used for the tilt $(\lambda)$ angles were used to define the phi $(\phi)$ angles $\left(e . g ., 49.85^{\circ}\right.$ for this structure, 2IAM V $\beta$, gold) by subtraction of $\lambda$ from a theoretical $180^{\circ}$ vector. This is illustrated with actual positions in 2IAM (A), showing the $180^{\circ}$ approximating, $176.32^{\circ}$ vector; the $\sim 90^{\circ}, 88.91^{\circ}$ angle; the $\sim 14.27 \AA, 14.14 \AA$ line segment; and the $\sim 16.90 \AA, 17.29 \AA$ line segment, i.e., to show the essential geometry within a structure; these are more accurately calculated by trigonometry in the derivation (B). Additionally, the rho $(\rho)$ segment from the appropriate $\alpha$-helix position (see $\lambda$ definitions) to a given $V$-domain's C22/23 was measured (here, $22.12 \AA$ ). The cone (full rotation) includes a probability (slice volume) through a scanning path $(d \theta)$ for CDR2. Figure $(A)$ is the original output of the PDB file as analysed in VMD-1.9.1. Figure (B) is a diagram constructed in MS-3D-paint. Best viewed at $100 \%$.

This is integration of a volume element in spherical coordinates (all $d V$ values in Tables 1A-1D). For compositional reasons throughout "the triple integral $d V$ " may be referred to as simply " $d V$." As discussed above it is not surprising that the $(\pi \cdot r / 4)$ values were close to the actual distances between CDR2 contacts in the aforementioned MHC $\alpha$-helix ranges using this approach, e.g., $13.27 \AA$, Figure 2B. Thus, the volume of the cone slice for each $\mathrm{V}$-domain was determined upon integration for the three spherical coordinates, rho $(\rho)$, theta $(\theta)$ and phi $(\phi)$, where the upper limit of each integral is derived from the measured $\rho$ and $\phi$ values of each $\mathrm{V}$ domain as shown:

$$
\iiint_{V} d V=\int_{0}^{\sin ^{-1}(r / \rho)} \int_{0}^{\pi \cdot r / 4} \int_{0}^{z / \cos \phi} \rho^{2} \sin \phi d \rho d \theta d \phi
$$

Since $\rho$ and $\phi$ are measured for each $\vee$ domain, derivations of upper limits for the first and third integrals simply provide trigonometric relationships. 
Table 1A Summary Geometry TCR:pHLA-DR

\begin{tabular}{|c|c|c|c|c|c|c|c|c|c|}
\hline PDB & $\mathrm{CDR} 2^{1}$ & CDR3 & $\sigma \uparrow$ & $\lambda$ & $\omega$ & $\varphi$ & $k \neq$ & $\rho(\AA)^{0}$ & $d V^{\#}$ \\
\hline $2 \mathrm{AMM} \alpha *$ & $\begin{array}{c}\text { K53NZ:E69OE2 } \\
2.77\end{array}$ & $\begin{array}{c}\text { G920:G58N } \\
3.34\end{array}$ & 50.75 & 116.86 & 101.05 & $\begin{array}{l}30.73 c \\
18.13 m\end{array}$ & 1.69 & 29.55 & 1703 \\
\hline 2IAMB & $\begin{array}{c}\text { V50CG } 2: 057 \mathrm{CB} \\
3.96\end{array}$ & $\begin{array}{c}\text { T980G1:D660D2 } \\
2.53\end{array}$ & 30.97 & 130.15 & 66.21 & $\begin{array}{l}12.73 \mathrm{c} \\
17.69 \mathrm{~m}\end{array}$ & 0.719 & 22.12 & 297 \\
\hline $2 \mathrm{IAN} \alpha$ & $\begin{array}{c}\text { K53NZ:E69OE1 } \\
2.95\end{array}$ & $\begin{array}{c}\text { A93N:E55O } \\
3.51\end{array}$ & 50.99 & 116.16 & 99.72 & $\begin{array}{l}30.42 c \\
19.32 m\end{array}$ & 1.57 & 29.39 & 1710 \\
\hline 2IANB & $\begin{array}{c}\text { Y48OH:Q57NE2 } \\
3.30\end{array}$ & $\begin{array}{c}\text { T980G1:D660D1 } \\
2.74\end{array}$ & 30.27 & 130.76 & 64.98 & $\begin{array}{l}12.21 \mathrm{c} \\
29.69 \mathrm{~m}\end{array}$ & 0.411 & 21.78 & 270 \\
\hline $4 \mathrm{E} 41 \alpha$ & $\begin{array}{c}\text { K53NZ:E69OE2 } \\
2.63\end{array}$ & $\begin{array}{c}\text { T93OG1:E55OE1 } \\
3.17\end{array}$ & 48.39 & 119.79 & 100.80 & $\begin{array}{l}29.00 \mathrm{c} \\
16.93 \mathrm{~m}\end{array}$ & 1.71 & 29.51 & 1512 \\
\hline $4 E 41 \beta$ & $\begin{array}{c}\text { N54ND2:E55OE2 } \\
3.24\end{array}$ & $\begin{array}{c}\text { T970G1:D66OD2 } \\
2.51\end{array}$ & 32.08 & 129.37 & 65.97 & $\begin{array}{l}13.11 \mathrm{c} \\
26.55 \mathrm{~m}\end{array}$ & 0.494 & 23.67 & 406 \\
\hline
\end{tabular}

•same TCR (E8) and MHC (DRA1, DRB1*0104; pep 128) as 2ian (pep T28); same pMHC as 4E41 (TCR, G4).

${ }^{1}$ Contacts in angstroms $(\AA)$ measured with VMD software (www.ks. uiuc. edu); yellow highlighted contacts of the measured pitch angle $\left(\varphi_{m}\right)$, see text. Colour-coded possible H-bonds, pH 7: red (strong), magenta (medium), green (weakb hydrophobic(blue). The calculated pitch angle $\left(\varphi_{k}\right)$ of $V \alpha$ and $V \beta$ determined as before, using the formula:

$$
{ }^{\ddagger} \varphi_{c}=[\sigma \div(\sigma+\lambda)] \omega
$$

"Measured angles with VMD in degrees; "The value for $\rho$ as measured with VMD in $\mathcal{A}$; volume of the rotational density distribution (about $\mathrm{N}$-term $\mathrm{C} y$ s of the intra-domain disulfide) for $\mathrm{V} \alpha$ and $\mathrm{V} \beta$ was modeled as a cone (see text) where:

$$
\iiint_{V} d V=\int_{0}^{\sin ^{-1}(r / \rho)} \int_{0}^{\pi \cdot r / 4} \int_{0}^{z / \cos \phi} \rho^{2} \sin \phi d \rho d \theta d \phi
$$

And where, $\pi / 180$ is the conversion factor for the angular component of the integrand to yield $d V$ in $\AA^{3}$.

210

Two example solutions are given below (parameters as defined, Figs. 1 \& 2; text)-for all 38 triple integral solutions, see Suppl.1.I*. The $(\pi \cdot r / 4)$ circumference segment is used as the upper limit of the $d \theta$ integral because it is accurate to a path, i.e., a distance; formally (for $\theta$ angle in degrees): $\pi \cdot r(\theta) / 180=\operatorname{arc~length~in~} \AA$ [22]. Hence, $d \phi$ is the only integrand for conversion (multiplying by $\pi \div 180$ ) to yield the cubic angstrom $\left(\AA^{3}\right)$ unit of volume. The C22/23 cysteine is historically used as the center of any given $\mathrm{V}$ domain [10] and each $\mathrm{V}$-domain coneslice is thus the volumetric-density through its CDR2 scanning path (e.g., Fig. 2B). The calculus interprets a comprehensive geometry of the $\mathrm{V}$ domain into a probability of scanning using only the ground-state structure, i.e., without crystallographic data on theoretical conformerssuggesting here, a classic protein function, allostery [24]. 
Table 1B Summary Geometry TCR:pHLA-DR

\begin{tabular}{|c|c|c|c|c|c|c|c|c|c|}
\hline PDB & $\mathrm{CDR}^{1}{ }^{1}$ & CDR3 & $\sigma^{\uparrow}$ & $\lambda$ & $\omega$ & $\varphi$ & $k \neq$ & $\rho(\AA)^{0}$ & $d V^{¥}$ \\
\hline $1 \mathrm{H} 8 \mathrm{H}^{\dagger}$ & $\begin{array}{c}\text { A52CB:E69CD } \\
3.54\end{array}$ & $\begin{array}{c}\text { E102CD:A61CB } \\
4.07\end{array}$ & 35.67 & 134.93 & 86.88 & $\begin{array}{l}18.17 \mathrm{c} \\
17.15 \mathrm{~m}\end{array}$ & 1.06 & 29.77 & 740 \\
\hline $1 \mathrm{~J} 8 \mathrm{H} \beta$ & $\begin{array}{c}\text { D510D1:K67NZ } \\
2.54 \\
\end{array}$ & $\begin{array}{c}\text { P100CG:L67CD1 } \\
3.43 \\
\end{array}$ & 47.99 & 109.62 & 84.91 & $\begin{array}{r}25.85 c \\
25.95 m\end{array}$ & 0.996 & 27.91 & 1734 \\
\hline $1 \mathrm{FYT} \alpha$ & $\begin{array}{c}\mathrm{A} 52 \mathrm{CB}: \mathrm{E} 69 \mathrm{CD} \\
4.42\end{array}$ & $\begin{array}{c}\text { K103NZ:057OE1 } \\
4.36\end{array}$ & 37.11 & 133.00 & 86.29 & $\begin{array}{l}18.81 \mathrm{c} \\
16.90 \mathrm{~m}\end{array}$ & 1.11 & 29.70 & 826 \\
\hline 1 FYT $\beta$ & $\begin{array}{c}\text { D510D1:K67NZ } \\
2.67 \\
\end{array}$ & $\begin{array}{c}\text { P100CB:D66CB } \\
4.96 \\
\end{array}$ & 47.87 & 109.97 & 85.82 & $\begin{array}{c}26.03 c \\
23.44 m\end{array}$ & 1.11 & 28.04 & 1747 \\
\hline 3Т0Е $\alpha$ & $\begin{array}{c}\text { L50CD1:T106CG2 } \\
3.65 \\
\end{array}$ & $\begin{array}{c}\text { K96NZ:Q57OE1 } \\
4.06\end{array}$ & 38.06 & 132.31 & 88.95 & $\begin{array}{c}19.87 \mathrm{c} \\
21.36 \mathrm{~m}\end{array}$ & 0.930 & 29.99 & 855 \\
\hline $3 \mathrm{TOE} \beta^{*}$ & $\begin{array}{c}\text { E500E1-K67NZ } \\
3.77\end{array}$ & $\begin{array}{c}\text { N100CB:Q99CD } \\
4.08 \\
\end{array}$ & 44.41 & 110.49 & 76.75 & $\begin{array}{r}22.00 \mathrm{c} \\
20.98 \mathrm{~m}\end{array}$ & 1.05 & 24.63 & 1024 \\
\hline $4 \mathrm{H} 1 \mathrm{~L} \alpha$ & $\begin{array}{c}\text { F50CD2:Q70CG } \\
3.17\end{array}$ & $\begin{array}{c}\text { T980G1:E55OE2 } \\
4.32 \\
\end{array}$ & 44.40 & 125.30 & 94.81 & $\begin{array}{c}24.75 \mathrm{c} \\
19.78 \mathrm{~m}\end{array}$ & 1.25 & 32.62 & 1782 \\
\hline $4 \mathrm{H} 1 \mathrm{~L} \beta$ & $\begin{array}{c}149 C D 1: L 60 C B \\
3.30\end{array}$ & $\begin{array}{c}\text { Y97CZ:D66CB } \\
3.77\end{array}$ & 41.83 & 117.27 & 75.97 & $\begin{array}{c}19.98 \mathrm{c} \\
18.84 \mathrm{~m}\end{array}$ & 1.06 & 27.16 & 1198 \\
\hline $1 Z G L \alpha$ & $\begin{array}{c}\text { K51NZ:D760D1 } \\
6.91 \\
\end{array}$ & $\begin{array}{c}\text { S91OG:E55OE2 } \\
2.90\end{array}$ & 51.82 & 117.60 & 85.36 & $\begin{array}{l}26.11 \mathrm{c} \\
22.11 \mathrm{~m}\end{array}$ & 1.18 & 33.78 & 2831 \\
\hline $1 Z G L \beta$ & $\begin{array}{c}\text { N51CG:A64CB } \\
3.70\end{array}$ & $\begin{array}{c}\text { R99NH2:D66OD2 } \\
2.57 \\
\end{array}$ & 46.60 & 108.58 & 69.79 & $\begin{array}{l}20.96 \mathrm{c} \\
14.06 \mathrm{~m}\end{array}$ & 1.49 & 25.67 & 1281 \\
\hline
\end{tabular}

${ }^{\dagger}$ same TCR, peptide and DRA [DRB1-4] as 1fyt (DRB1*0104).

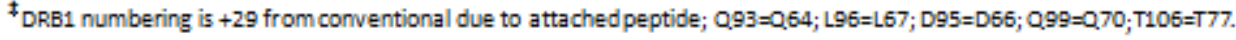

${ }^{1}$ Same as Table $1 \mathrm{~A}$ legend

223

Table 1C Summary Geometry TCR:pHLA-DR

\begin{tabular}{|c|c|c|c|c|c|c|c|c|c|}
\hline PDB & $\mathrm{CDR}^{1}{ }^{1}$ & CDR3 & $\sigma ף$ & $\lambda$ & $\omega$ & $\varphi$ & $k^{\neq}$ & $\rho(\stackrel{A}{A})^{0}$ & $d V^{\#}$ \\
\hline $6 \mathrm{CQL} \alpha^{\mathbb{1}}$ & $\begin{array}{c}\text { L58CD 1:A73CB } \\
3.85\end{array}$ & $\begin{array}{l}\text { A105N: E55OE1 } \\
3.00\end{array}$ & 37.14 & 133.17 & 93.59 & $\begin{array}{l}20.41 \mathrm{c} \\
23.24 \mathrm{~m}\end{array}$ & 0.878 & 29.01 & 746 \\
\hline $6 C Q L \beta$ & $\begin{array}{c}\text { S660G:Q57NE2 } \\
3.01\end{array}$ & $\begin{array}{c}\mathrm{R} 108 \mathrm{NH} 2: \mathrm{Y} 600 \mathrm{H} \\
2.93\end{array}$ & 32.68 & 129.70 & 70.67 & $\begin{array}{l}14.22 \mathrm{c} \\
18.95 \mathrm{~m}\end{array}$ & 0.750 & 25.56 & 542 \\
\hline 6CQNo & $\begin{array}{c}\text { L58CD2:T77CG2 } \\
3.73\end{array}$ & $\begin{array}{l}\text { A105N: E55OE1 } \\
2.89\end{array}$ & 36.31 & 134.45 & 92.61 & $\begin{array}{l}19.69 c \\
22.34 m\end{array}$ & 0.881 & 28.98 & 690 \\
\hline $6 \mathrm{CQNB}$ & $\begin{array}{c}\text { S660G:Q57NE2 } \\
3.17\end{array}$ & $\begin{array}{c}\text { L109CD1:D66CB } \\
3.73 \\
\end{array}$ & 33.40 & 128.57 & 70.09 & $\begin{array}{l}14.45 c \\
23.56 m\end{array}$ & 0.613 & 25.68 & 586 \\
\hline $6 \mathrm{CQQ} \alpha$ & $\begin{array}{c}\mathrm{L58CD} 2: \mathrm{A} 73 \mathrm{CB} \\
3.78\end{array}$ & $\begin{array}{l}\text { A105N: E55OE1 } \\
2.88\end{array}$ & 35.95 & 134.32 & 89.29 & $\begin{array}{l}18.85 c \\
24.02 m\end{array}$ & 0.785 & 28.97 & 693 \\
\hline $6 \mathrm{CQQ} \beta$ & $\begin{array}{c}\text { S660G:Q57OE1 } \\
3.64\end{array}$ & $\begin{array}{c}\mathrm{R} 108 \mathrm{NH} 2: \mathrm{Y} 600 \mathrm{H} \\
2.97\end{array}$ & 34.88 & 127.12 & 72.65 & $\begin{array}{l}15.64 \mathrm{c} \\
22.76 \mathrm{~m}\end{array}$ & 0.687 & 26.34 & 800 \\
\hline $6 \mathrm{CQR} \alpha$ & $\begin{array}{c}\text { T57OG1:Q7ONE2 } \\
3.17\end{array}$ & $\begin{array}{l}\text { A105N:E55OE1 } \\
3.03\end{array}$ & 36.52 & 133.75 & 87.44 & $\begin{array}{l}18.75 \mathrm{c} \\
23.44 \mathrm{~m}\end{array}$ & 0.800 & 28.99 & 718 \\
\hline $6 \mathrm{CQR} \beta$ & $\begin{array}{c}\text { E600E2: K67NZ } \\
3.07\end{array}$ & $\begin{array}{c}\mathrm{R} 108 \mathrm{NH} 2: \mathrm{Y} 600 \mathrm{H} \\
3.11\end{array}$ & 36.34 & 124.36 & 69.70 & $\begin{array}{l}15.76 \mathrm{c} \\
29.07 \mathrm{~m}\end{array}$ & 0.542 & 25.95 & 746 \\
\hline
\end{tabular}

" SameTCR (F24) and peptide and DRA (DRB1-11) as 6Cqq (DRB1-15) and 6Cqr (DRB1-1); same pMHC as 6cqn (TCR, F5; CDR3ß G108R). 
Table 1D Summary of TCR:pHLA-DQ Geometry

\begin{tabular}{|c|c|c|c|c|c|c|c|c|c|}
\hline PDB & $\mathrm{CDR}^{1}{ }^{1}$ & CDR3̣ & $\sigma^{\pi}$ & $\lambda$ & $\omega$ & $\varphi$ & $k^{\ddagger}$ & $\rho^{0}$ & $d V^{\#}$ \\
\hline $5 \mathrm{KSA}^{\dagger}{ }^{\dagger}$ & $\begin{array}{c}\text { Y57CE1:R70CG } \\
3.71\end{array}$ & $\begin{array}{l}\text { Y1130H:D550D2 } \\
2.64\end{array}$ & 36.16 & 134.95 & 94.04 & $\begin{array}{l}15.90 \mathrm{c} \\
19.87 \mathrm{~m}\end{array}$ & 0.800 & 28.76 & 651 \\
\hline $5 \mathrm{KSA} \beta$ & $\begin{array}{c}\text { Y57CE2:A64CB } \\
4.13\end{array}$ & $\begin{array}{c}\text { Y114CD1:E66CG } \\
3.59\end{array}$ & 40.26 & 119.15 & 71.38 & $\begin{array}{l}18.03 c \\
23.32 m\end{array}$ & 0.773 & 26.02 & 939 \\
\hline $5 \mathrm{KS} 9 \alpha$ & $\begin{array}{c}\text { S580G:D760D2 } \\
2.65\end{array}$ & $\begin{array}{c}\text { N114ND2:T610G1 } \\
4.24\end{array}$ & 41.63 & 128.32 & 92.51 & $\begin{array}{l}22.66 \mathrm{c} \\
16.96 \mathrm{~m}\end{array}$ & 1.34 & 28.65 & 918 \\
\hline $5 \mathrm{KS} 9 \beta$ & $\begin{array}{c}\text { R66NE:057OE1 } \\
3.14\end{array}$ & $\begin{array}{c}\text { D114OD2:E66OE2 } \\
3.67\end{array}$ & 33.80 & 126.25 & 67.09 & $\begin{array}{c}14.17 \mathrm{c} \\
18.03 \mathrm{~m}\end{array}$ & 0.786 & 23.57 & 465 \\
\hline $4 \mathrm{OZF} \alpha^{\ddagger}$ & $\begin{array}{c}\text { H55NE2:R7ONH2 } \\
2.89\end{array}$ & $\begin{array}{c}\text { A113CB:F58C2 } \\
5.05\end{array}$ & 40.39 & 131.50 & 111.69 & $\begin{array}{c}26.24 c \\
29.44 m\end{array}$ & 0.891 & 33.17 & 1397 \\
\hline $4 O Z F \beta$ & $\begin{array}{c}\text { N63OD1:KG7NZ } \\
3.50\end{array}$ & $\begin{array}{c}\text { D114OD1:R70NE } \\
3.73\end{array}$ & 37.47 & 120.69 & 66.87 & $\begin{array}{c}15.84 c \\
25.68 \mathrm{~m}\end{array}$ & 0.617 & 23.16 & 554 \\
\hline $4 \mathrm{OZG} \alpha$ & $\begin{array}{c}\text { H55NE2:E690E1 } \\
2.93\end{array}$ & $\begin{array}{c}\text { A115CB:057CB } \\
4.11\end{array}$ & 45.55 & 124.99 & 100.43 & $\begin{array}{c}26.82 c \\
26.64 m\end{array}$ & 1.01 & 31.91 & 1657 \\
\hline $4 O Z G \beta$ & $\begin{array}{c}\text { Y55OH:Q570E1 } \\
3.35\end{array}$ & $\begin{array}{c}\text { Y117OH:D660D2 } \\
2.72\end{array}$ & 30.93 & 128.29 & 61.35 & $\begin{array}{c}11.92 \mathrm{c} \\
31.10 \mathrm{~m}\end{array}$ & 0.383 & 21.22 & 276 \\
\hline $40 Z \mathrm{H} \alpha$ & $\begin{array}{l}\text { H55NE2:E69OE1 } \\
2.79\end{array}$ & $\begin{array}{c}\mathrm{A} 110 \mathrm{CB}: \mathrm{D} 55 \mathrm{CB} \\
3.83\end{array}$ & 48.60 & 121.57 & 102.66 & $\begin{array}{c}29.32 c \\
26.64 m\end{array}$ & 1.10 & 32.35 & 2030 \\
\hline $4 \mathrm{OZH} \beta$ & $\begin{array}{c}\text { Y55OH:Q570E } 1 \\
2.86\end{array}$ & $\begin{array}{c}\text { Y117OH:D660D2 } \\
3.21\end{array}$ & 29.94 & 130.19 & 61.67 & $\begin{array}{c}11.53 \mathrm{c} \\
32.11 \mathrm{~m}\end{array}$ & 0.359 & 21.47 & 267 \\
\hline $4 \mathrm{OZI} \alpha$ & $\begin{array}{c}\text { Q55OE1:R7ONH1 } \\
2.78 \\
\end{array}$ & $\begin{array}{c}\text { S111OG:D55OD1 } \\
3.21\end{array}$ & 44.59 & 125.38 & 110.06 & $\begin{array}{c}28.87 \mathrm{c} \\
19.13 \mathrm{~m}\end{array}$ & 1.51 & 31.72 & 1588 \\
\hline $4 O Z I \beta$ & $\begin{array}{c}\text { T660G1:0570E1 } \\
4.08 \\
\end{array}$ & $\begin{array}{c}\text { Y115OH:D660D1 } \\
2.56\end{array}$ & 38.48 & 116.06 & 66.45 & $\begin{array}{c}16.55 c \\
23.76 \mathrm{~m}\end{array}$ & 0.700 & 22.10 & 549 \\
\hline $4 G G 6 \alpha$ & $\begin{array}{c}\qquad 57 \mathrm{CD} 2: \mathrm{A} 7 \mathrm{CB} \\
3.98\end{array}$ & $\begin{array}{c}\text { R110CD:F58CD1 } \\
3.71\end{array}$ & 38.77 & 132.70 & 98.93 & $\begin{array}{l}22.37 \mathrm{c} \\
25.38 \mathrm{~m}\end{array}$ & 0.881 & 30.87 & 982 \\
\hline $4 G G 6 \beta$ & $\begin{array}{c}\text { N58ND2:H68ND1 } \\
3.94\end{array}$ & $\begin{array}{c}\text { T113OG1:R7ONE } \\
2.95\end{array}$ & 35.96 & 124.32 & 68.59 & $\begin{array}{c}15.39 \mathrm{c} \\
22.73 \mathrm{~m}\end{array}$ & 0.677 & 25.02 & 645 \\
\hline
\end{tabular}

${ }^{\dagger}$ Bel602 TCR same germline (TRAV 20*01/TRBV 9*01) as $5 \mathrm{ks} 9$ (Bel502); MHC (DQ8.5) different from $5 \mathrm{ks} 9$ (DQ8); peptide different [glia-gamma-1 (1-11)] from $5 \mathrm{ks} 9$ [glia-alpha-1 (1-16)].

${ }^{\ddagger}$ Same MHC (DQ2) and peptide [glia-alpha-2 (1-13)] as 4ozg and 4ozh; 4ozi has same MHC (DQ2) with different peptide [glia-alpha-1 (1-13)]; all have different TCR.

१ी Same MHC (DQ8) as $5 \mathrm{ks} 9$.

${ }^{1}$ referto Table 1 A legend.

Accordingly, the mean $d V$ of these TCR V-domains was $955 \AA^{3}$, excluding the unusually large $d V$ of the 1ZGL $V \alpha$. Importantly, with the exclusion of 1ZGL (an apparent outlier) there is a linear relationship between calculated pitch $\left(\varphi_{c}\right)$ and the $d V$ triple integral (see plotted values from Tables 1A-1D in the Suppl.1.II) - corresponding to the equation: $y=83.60 x-719.40$; where $R^{2}$ $\approx 0.900$, by linear regression analysis. The lowest $d V$ structures have the lowest calculated pitch values. Overall, it says something about TCR:pMHC that may not be intuitive-that a "flush" Vdomain geometry could limit CDR2 "finding" an optimal binding interface with side-chains involving the aforementioned $\alpha$-helix regions. This is the clonotypic nature of TCR function.

\section{CDR3:pMHC Contacts}


239

240

241

242

243

244

245

246

247

248

249

250

All these structures, even those with the highest resolution, are subject to limitations of crystallography; and indeed, computed $\mathrm{H}$-bonds are based on these coordinates. Nevertheless, a correlation between relative $d V$ and contact distances for hydrogen bonds of the five components could be investigated; see Tables $2 \mathrm{~A}-2 \mathrm{C}$. For example, asking if particularly strong (close) $\mathrm{H}$-bonds are linked to a restricted $d V$ ?

2IAM-V $\alpha$

$$
\begin{aligned}
& \lambda=116.86^{\circ} \quad \rho=29.55 \AA \\
& \phi=180-116.86=63.14^{\circ} \quad r=\sin \phi(\rho)=0.892(29.55)=26.36 \AA \\
& \pi \cdot r / 4=3.1 \overline{4}(26.36) / 4=20.70 \AA \\
& \iiint_{V} d V=\int_{0}^{63.14} \int_{0}^{20.70} \int_{0}^{29.55} \rho^{2} \sin \phi d \rho d \theta d \phi \quad=\int_{0}^{63.14} 20.70\left[\frac{(29.55)^{3}}{3} \sin \phi\right] d \phi \quad \text { where, } \int \sin \phi=-\cos \phi \\
& =\left.\int_{0}^{63.14} \int_{0}^{20.70} \frac{1}{3} \rho^{3} \sin \phi\right|_{\rho=0} ^{\rho=29.55} d \theta d \phi \quad=-\left.20.70\left[\frac{(29.55)^{3}}{3}\right] \cos \phi\right|_{\phi=0} ^{\phi=63.14} \text { where, } \cos 0=1 \\
& =\int_{0}^{63.14} \int_{0}^{20.70} \frac{(29.55)^{3}}{3} \sin \phi d \theta d \phi \quad=.452\left[-20.70\left[\frac{(29.55)^{3}}{3}\right]\right]-1\left[-20.70\left[\frac{(29.55)^{3}}{3}\right]\right]
\end{aligned}
$$

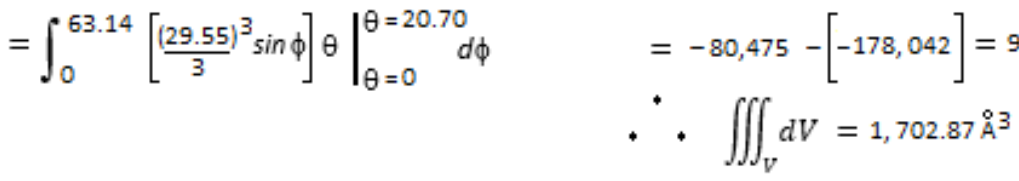

2IAM-V $\beta$

$$
\begin{aligned}
& \lambda=130.15^{\circ} \quad \rho=22.12 \AA \\
& \phi=180-130.15=49.85^{\circ} \quad r=\sin \phi(\rho)=0.764(22.12)=16.90 \AA \\
& \pi \cdot r / 4=3.1 \overline{4}(16.90) / 4=13.27 \AA \\
& \left.\iiint_{V} d V=\int_{0}^{49.85} \int_{0}^{13.27} \int_{0}^{22.12} \rho^{2} \sin \phi d \rho d \theta d \phi \quad=\int_{0}^{49.85} 13.27\left[\frac{(22.12}{3}\right)^{3} \sin \phi\right] d \phi \quad \text { where, } \int \sin \phi=-\cos \phi \\
& \left.=\left.\int_{0}^{49.85} \int_{0}^{13.27} \frac{1}{3} \rho^{3} \sin \phi\right|_{\rho=0} ^{\rho=22.12} d \theta d \phi \quad=-13.27\left[\frac{(22.12}{3}\right)^{3}\right]\left.\cos \phi\right|_{\phi=0} ^{\phi=49.85} \text { where, } \cos 0=1 \\
& =\int_{0}^{49.85} \int_{0}^{13.27} \frac{(22.12)^{3}}{3} \sin \phi d \theta d \phi \quad=.645\left[-13.27\left[\frac{(22.12)^{3}}{3}\right]\right]-1\left[-13.27\left[\frac{(22.12)^{3}}{3}\right]\right] \\
& =\left.\int_{0}^{49.85}\left[\frac{(22.12)^{3}}{3} \sin \phi\right] \theta\right|_{\theta=0} ^{\theta=13.27} d \phi \quad=-30,879-[-47,875]=16,996 \quad \text { where, multipl. } \times \frac{\pi}{180} \text { corrects for angular integrand of } \phi \\
& \text { * * } \iiint_{V} d V=296.64 \AA^{3}
\end{aligned}
$$

*for all $(n=38)$ triple-integral solutions, see Suppl.1.I. 
Table 2A Possible H-bonding networks in TCR:pHLA-DR complexes

\begin{tabular}{|c|c|c|c|c|c|c|c|}
\hline PDB & CDR3a:pep & Nn MHC.pep ${ }^{5}$ & CDR3ßpep & pep:pep & $\alpha: \alpha, \alpha: \beta, \beta: \beta$ & MHC:MHC & $\alpha / \mathrm{M}: \mathrm{MHC}$ \\
\hline 2IAM* & none & $\begin{array}{c}\text { A1N62ND2:L290 } \\
2.0 \\
\text { A31N:A1N62OD2 } \\
2.2 \\
\text { A1N69ND2:A320 } \\
2.1 \\
\text { V34N:A1N69OD1 } \\
1.8 \\
\text { B1R71NH1:N300 } \\
2.1 \\
\text { B1R71NHZ2:N300 } \\
2.0 \\
\text { NGOND2:B1QT0OE1 } \\
2.4\end{array}$ & $\begin{array}{c}\text { T95OH:A510 } \\
17 \\
\text { H96NE2:N300 } \\
2.8 \\
\text { H96NE2:A310 } \\
2.8\end{array}$ & none & 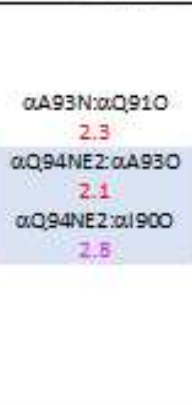 & $\begin{array}{c}\text { B1R71NH1: } \\
\text { Q70OE1 } \\
2.2\end{array}$ & $\begin{array}{c}\text { QQGINE2:B1T770 } \\
2.2 \\
\text { A1G5BN:aG920 } \\
2.4 \\
\text { BT9BN:B1D66OD1 } \\
2.0\end{array}$ \\
\hline $\begin{array}{l}\text { 2IAN } \\
\text { [no H's) }\end{array}$ & none & $\begin{array}{c}\text { A1N52ND2:L290 } \\
3.0 \\
\text { A1N69ND2:A320 } \\
3.1 \\
\text { V34N:A1N69OD1 } \\
3.0 \\
\text { B1R71NH1:N300 } \\
2.3 \\
\text { B1R71NH2:N300 } \\
2.5 \\
\end{array}$ & $\begin{array}{c}\text { T95OH:A310 } \\
2.5 \\
\text { H96NE2:N300 } \\
\quad: .8 \\
\text { H96NEZ:A310 } \\
3.8 \\
\end{array}$ & none & $\begin{array}{c}\text { aG92N:a.9CO } \\
3.3\end{array}$ & $\begin{array}{c}\text { B1R71NH1: } \\
\text { Q700E1 } \\
3.2\end{array}$ & $\begin{array}{c}\text { aQ91NE2:B1T770 } \\
2.9 \\
\text { PT9BOG1:D66001 } \\
2.7\end{array}$ \\
\hline 4E41 & $\begin{array}{c}128 \mathrm{~N}: 6910 \\
1.9\end{array}$ & $\begin{array}{c}\text { A31N:A1NG2OD1 } \\
1.7 \\
\text { A1NG2ND2:1290 } \\
2.0 \\
\text { A1N69ND2:A320 } \\
2.1 \\
\text { V34N:A1N69OD1 } \\
2.0 \\
\text { B1R71NH1:N300D1 } \\
2.1 \\
\text { B1R71NH2:N300 } \\
1.8 \\
\text { B1Q7ONE2:N300 } \\
2.8 \\
\end{array}$ & $\begin{array}{c}\text { R95NH1:1280 } \\
19\end{array}$ & none & $\begin{array}{c}\text { BR95NH2:0.6910 } \\
2.0 \\
\text { PR95NHZZ:aT930 } \\
2.4 \\
\text { BR95NE:0.DB9OD1 } \\
2.5\end{array}$ & none & $\begin{array}{c}B T 97 N: D E 6 O D 2 \\
1.9\end{array}$ \\
\hline
\end{tabular}

${ }^{5} \mathrm{Nn}$, nearest neighbour; shown possible H-bonds, donor $\rightarrow$ acceptor; colour-code: $s 2.5$, red; 2.6 to 2.9 , mauve; 23.0 , green.

- Same TCR (E8) and MHC (DRA, DRB1*0104; pep 128) as 2ian (pep T28); same pMHC as 4E41 (TCR, G4). 
Table 2B Possible H-bonding networks in TCR:pHLA-DR complexes

\begin{tabular}{|c|c|c|c|c|c|c|c|}
\hline$P D B$ & CDR3 3 :pep & Nn MHC pep ${ }^{5}$ & CDR3ß:pep & pep:pep & $\alpha: \alpha, \alpha: \beta, \beta: \beta$ & MHC:MHC & $\mathrm{Nn} \propto \beta: \mathrm{MHC}$ \\
\hline $18 \mathrm{H}^{+}$ & none & $\begin{array}{c}\text { A1N62ND2:0,3110 } \\
1.9 \\
\text { A1N69ND2:L3140 } \\
1.8 \\
\text { L316N:A1N69OD1 } \\
1.7 \\
\text { B1Q7ONEZ:Q3110E } \\
1 \\
2.1 \\
\text { N312ND2:B1Q70OE } \\
1 \\
21\end{array}$ & $\begin{array}{c}\text { K315N:G9600 } \\
2.0\end{array}$ & none & 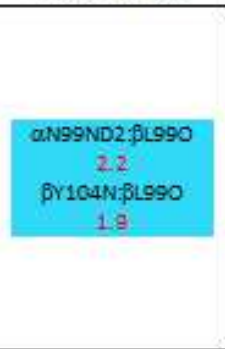 & none & none \\
\hline 1FYT & none & $\begin{array}{c}\text { A1N62ND2:03110 } \\
2.0 \\
\text { T313N:A1N62001 } \\
1.9 \\
\text { A1N69ND2:L3140 } \\
2.1 \\
\text { L316N:A1N690D1 } \\
1.7 \\
\text { B1R71NH1:N312OD } \\
1 \\
2.1 \\
\text { B1R71NH2:N3120 } \\
1.9 \\
\text { B1Q7ONEZ:Q311OE } \\
1 \\
2.3\end{array}$ & $\begin{array}{c}\text { N312ND2:T97O } \\
2.0 \\
\text { K315N:G9BO } \\
2.1\end{array}$ & $\begin{array}{l}\text { N312ND2:T31 } \\
30 \\
2.7 \\
\text { Q311NE2:K31 } \\
00 \\
2.5\end{array}$ & $\begin{array}{c}\text { ANG9ND2:81.990 } \\
2.0 \\
\text { PY104N:61990 } \\
2.1\end{array}$ & $\begin{array}{c}\text { B1R71NH1: } \\
\text { Q700E1 } \\
2.0\end{array}$ & none \\
\hline $3 \mathrm{TOE}^{*}$ & $\begin{array}{l}\text { A.5N: } 6920 \\
2.4\end{array}$ & $\begin{array}{c}\text { A1N62ND2:E60 } \\
2.4 \\
\text { R9NH1:B1Q930E1 } \\
2.3 \\
\text { R9NH1:B1D950D1 } \\
2.8 \\
\text { R9NH1:B1D95OD2 } \\
2.7\end{array}$ & $\begin{array}{c}\text { S9BN:Q80 } \\
2.5 \\
\text { R9NH2:N1000 } \\
2.2\end{array}$ & $\begin{array}{c}\text { G7N:E6OE1 } \\
2.6\end{array}$ & $\begin{array}{c}\text { BR95NH2:ON95OD1 } \\
1.8 \\
\text { PY99N:PS980G } \\
2.1\end{array}$ & $\begin{array}{c}\text { B1L96N: } \\
\text { D950D1 } \\
1.7\end{array}$ & $\begin{array}{c}\text { PS980G:A1NG2OD1 } \\
1.4 \\
\text { B1O99NE2:\$N1000 } \\
2.5\end{array}$ \\
\hline $4 \mathrm{H} 1 \mathrm{~L}$ & none & $\begin{array}{c}\text { A1NE2ND2:N40 } \\
19\end{array}$ & none & $\begin{array}{c}\text { C3SG:N4O } \\
2.5\end{array}$ & $\begin{array}{c}\text { aN97ND2:BT960G1 } \\
2.5\end{array}$ & none & $\begin{array}{c}\text { RR94NH2:A1N62OD1 } \\
16\end{array}$ \\
\hline $\begin{array}{l}12 G L \\
\left(n o H^{\prime} s\right)\end{array}$ & $\begin{array}{l}\text { VAN:D99001 } \\
\text { H2N:D99002 }\end{array}$ & $\begin{array}{c}\text { A1N62ND2:170 } \\
3: 2 \\
\text { N6ND2:A162OD1 } \\
3: 0\end{array}$ & none & none & 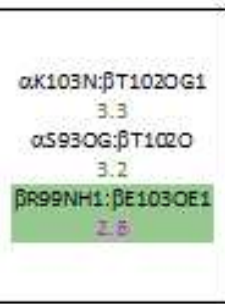 & none & 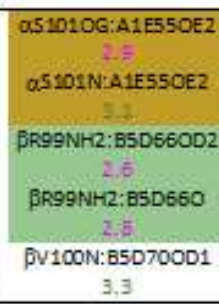 \\
\hline
\end{tabular}

${ }^{5} \mathrm{Nn}$, nearest neighbour; shown possible H-bonds, donor $\rightarrow$ acceptor; colour-code: $\leq 2.5$, red; 2.6 to 2.9 , mauve; 23.0 , green.

'Same TCR, peptide and DRA (DRB1-4) as 1fyt (DRB1*0104).

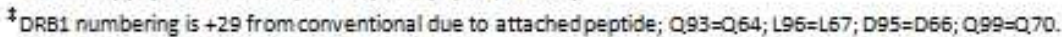

The structures were examined in Swiss-PDB Viewer/Deepview-v4.1 (www.wpdbv.vital-it.ch); in Swiss $\mathrm{H}$-bonds can be computed after hydrogens are added based on coordinates; complexity of certain structures (2IAN, 1ZGL) precluded computing hydrogens, and the shown $\mathrm{H}$-bonding distances are thus $\approx 1 \AA$ larger (Tables $2 \mathrm{~A}, 2 \mathrm{~B}$ ). Here, an obvious feature of all the complexes is that there are two principal foci of H-bonding; one involving N62 of DRA (A1) (shaded orange in the tables), which may include a separate secondary grouping at $\mathrm{N} 69$ (light-orange shade), and the DRB1 (B1) centre involving R71 (green shades). Note, R71 is polymorphic, so structures involving allotypes/isotypes that have a different a.a. at pos. $71(1 \mathrm{~J} 8 \mathrm{H}, 3 T 0 \mathrm{E}, 1 \mathrm{ZGL}$ 6CQQ \& $4 \mathrm{H} 1 \mathrm{~L})$ involve a different a.a. $(1 \mathrm{~J} 8 \mathrm{H}, 3 \mathrm{TOE}, 6 \mathrm{CQQ})$, or simply do not have a corresponding betachain $\mathrm{H}$-bonding centre $(4 \mathrm{H} 1 \mathrm{~L} \& 1 \mathrm{ZGL})$. Superimposed upon the $\mathrm{H}$-bonding centres are the $\mathrm{H}$ - 
bonds between the $\operatorname{CDR} 3 \alpha$ and peptide, the CDR3 $\beta$ and peptide, any peptide to peptide $\mathrm{H}$ bonding, any intra- or inter- bonds between the TCR V-domains, any $\mathrm{MHC}$ to $\mathrm{MHC} \mathrm{H}$-bonding, and direct $\mathrm{H}$-bonds between TCR V-domains and the MHC (Tables $2 \mathrm{~A}-2 \mathrm{C}$, columns).

Table 2C Possible H-bonding networks in TCR:pHLA-DR complexes

\begin{tabular}{|c|c|c|c|c|c|c|c|}
\hline$P D B$ & CDR3 $\alpha$ pep & Nn MHC pep ${ }^{5}$ & CDR3 B-pep & pep:pep & $\alpha=\alpha, \alpha, \beta, \beta=\beta$ & MHC:MHC & Nn $\alpha / \beta: M H C$ \\
\hline $6 \mathrm{CQL}^{\uparrow}$ & $\begin{array}{c}\text { R95NH1:A1050 } \\
2.0 \\
\text { R95NH1: } 61060 \\
2 . E \\
\text { R95NH1:N1070D1 } \\
2.3 \\
\text { R95NH2:N1070D1 } \\
2.0 \\
\end{array}$ & $\begin{array}{c}\text { A1N69ND2:E970 } \\
2.2 \\
\text { A99N:A1N690D1 } \\
1.5 \\
\text { B1R71NH1:R950 } \\
2.2 \\
\text { B1R71NH2:R950 } \\
2.0 \\
\end{array}$ & $\begin{array}{c}\text { A11ON:E97OE1 } \\
1.9 \\
\text { G111N:E97OE2 } \\
1.9\end{array}$ & $\begin{array}{c}\text { Q9EN:E97OE1 } \\
2.0\end{array}$ & $\begin{array}{c}\text { BG112N:PL109O } \\
2.7\end{array}$ & $\begin{array}{c}\text { B1R71NH2: } \\
\text { D70002 } \\
2.4\end{array}$ & $\begin{array}{l}\text { OA105N:A1E55OE1 } \\
2.1\end{array}$ \\
\hline $6 \mathrm{CQN}$ & $\begin{array}{c}\text { R95NH1:A1050 } \\
2.1 \\
\text { R95NH1:G1060 } \\
2.5 \\
\text { R95NH1:N1070D1 } \\
2.2 \\
\text { R95NH2:N1070D1 } \\
1.9\end{array}$ & $\begin{array}{c}\text { A96N:A1N62OD1 } \\
1.9 \\
\text { A1N62ND2:L940 } \\
1.9 \\
\text { A1N69ND2:E970 } \\
2.2 \\
\text { A99N:A1N69OD1 } \\
1.8 \\
\text { B1R71NH1:R950 } \\
2.0 \\
\text { B1R71NH2:R950 } \\
1.9 \\
\end{array}$ & $\begin{array}{c}\text { A11ON:E97OE1 } \\
17 \\
\text { G111N:E97OE2 } \\
20\end{array}$ & $\begin{array}{c}\text { Q9EN:E97OE1 } \\
2.2\end{array}$ & $\begin{array}{c}\text { BG112N:PL109O } \\
2.5\end{array}$ & $\begin{array}{c}\text { B1R71NH2: } \\
\text { D7DOD2 } \\
1.9\end{array}$ & $\begin{array}{c}\text { OA1OSN:A1E55OE } 1 \\
2.0\end{array}$ \\
\hline $6 \mathrm{CQQ}$ & $\begin{array}{c}\text { R95NH1:G1060 } \\
2.4 \\
\text { R95NH1:N1070D1 } \\
2.3 \\
\text { R95NHZ:N1070D1 } \\
1.8\end{array}$ & $\begin{array}{c}\text { A96N:A1NS2OD1 } \\
1.5 \\
\text { A1N62ND2:L94O } \\
1.3 \\
\text { A1N69ND2:E970 } \\
2.1 \\
\text { A99N:A1N69OD1 } \\
2.0 \\
\text { 5100N:B1D57OD1 } \\
2.5\end{array}$ & $\begin{array}{l}\text { R95NH2:A1100 } \\
2.0 \\
\text { A110N:E97OE1 } \\
2.1 \\
\text { G111N:E97OE2 } \\
2.3\end{array}$ & $\begin{array}{c}\text { Q98BN:E97OEI } \\
2.2\end{array}$ & $\begin{array}{c}\text { BQ115NE2: CON1140 } \\
2.1\end{array}$ & none & $\begin{array}{l}\text { OA1OSN:A1E55OE1 } \\
2,0 \\
\begin{array}{c}\text { BR1OBNH2:B1D66OD1 } \\
2,2\end{array} \\
\text { BR10BNH2:B1D66OD2 } \\
2.8\end{array}$ \\
\hline 6CQR & $\begin{array}{c}\text { R95NH1:A1050 } \\
2.2 \\
\text { R95NH1:N107001 } \\
2.1 \\
\text { R95NH2:N107001 } \\
2.0\end{array}$ & $\begin{array}{c}\text { A96N:A1N62001 } \\
1.9 \\
\text { A1N62ND2:L940 } \\
2.1 \\
\text { A1N69ND2:E970 } \\
2.2 \\
\text { A99N:A1N69OD1 } \\
1.5 \\
\text { B1R71NH1:R950 } \\
2.4 \\
\text { B1R71NH2:R950 } \\
1.5\end{array}$ & $\begin{array}{c}\text { R95NH2:A1100 } \\
2.2 \\
\text { A110N:E970E1 } \\
1.8 \\
\text { G111N:E97OE2 } \\
2.2\end{array}$ & $\begin{array}{c}\text { Q9EN:E97OE1 } \\
24\end{array}$ & $\begin{array}{c}\text { BQ116NE2:ON114O } \\
2,1\end{array}$ & none & $\begin{array}{c}\text { OA1OSN:A1E55OE } 1 \\
2.0\end{array}$ \\
\hline
\end{tabular}

${ }^{5} \mathrm{Nn}$, nearest neighbour; shown possible H-bonds, donor $\rightarrow$ acceptor; colour-code: $\leq 2.5$, red; 2.6 to 2.9 , mauve; 23.0 , green.

15ame TCR \{F24) andpeptide and DRA (DRB1-11) as 6cqq (DRB1-15) and 6cqr (DRB1-1); same pMHC as 6cqn (TCR, F5; CDR3 B G10BR).

In all cases, the CDR3 $\alpha$ :peptide bonds are either non-existent, or isolated from the MHC:peptide centres. In 4E41, 6CQQ and 6CQR, CDR3 $\alpha$ may link to CDR3 $\beta$ through both bonding nearby with peptide (blue shading). For $2 \mathrm{IAM}, 1 \mathrm{~J} 8 \mathrm{H}$, and $1 \mathrm{FYT}$ there are bonds between intra-V (2IAM), or intra- and inter-V (1J8H, 1FYT), but these are not linked to the peptide (different blue shade). N62 is connected to a peptide:peptide $\mathrm{H}$-bond (4H1L), or to an inter-V $\beta$ bond (3TOE); in both cases these potential networks extend to the $\mathrm{V} \beta$ directly binding with N62. 1ZGL is the only structure with an inter-V bond potentially linked to a direct Vdomain interaction with $\mathrm{MHC}$, while $4 \mathrm{H} 1 \mathrm{~L}$ is unique in not showing any $\mathrm{DRB} \mathrm{H}$-bonding involving the CDR3 (these two structures involve non-DRB1 isotypes). In 6CQL and 6CQN there is a potential DRB1:peptide link with an MHC:MHC bond, but this does not involve the TCR. The 
6CQ series all have CDR3 $\beta$ :peptide bonds also involving a peptide:peptide bond; this is not found in any of the other complexes (mauve shading). Finally, and most significantly here, in 2IAM/2IAN, 1FYT and 3TOE the DRB1:peptide centre is potentially connected through a CDR3 3 :peptide bond with an MHC:MHC H-bond (Table 2A \& 2B, green shades).

\section{CDR3:pMHC Chemistry}

$\mathrm{H}$-bonds computed in Swiss are potential $\mathrm{H}$-bonds, and to assess a given network the chemistry of each bond must be examined $[24,25]$. The analyses of Tables $2 A-2 C$ indicated that a potential $\mathrm{H}$-bonding network where CDR3 $\beta$ is connected through peptide-bonding to DRB1:peptide and DRB1:DRB1 $\mathrm{H}$-bonds might correlate with the highly-restricted $d V$ of $V \beta$ in 2IAM $\left(297 \AA^{3}\right)$ and 2IAN $\left(270 \AA^{3}\right)$. However, these networks would need to be distinct from this same type of potential network observed in 1FYT and 3TOE (Table 2B), which have $d V$ for $V \beta$ of $1747 \AA^{3}$ and $1024 \AA^{3}$, respectively (Table $1 B$ ). Shown is the analysis of the 2IAM-V $\beta$ versus $1 F Y T-V \beta$ for contacts made by each CDR3 $\beta$ (Fig. 3). For 2IAM-V $\beta$ a potential $H$-bonding network involving CDR3 $\beta$ a.a. Y95 and H96 focused on central peptide a.a. N30 and A31 is apparent; and DRB1 a.a. Q70 and R71 appear in this same network, while the $\beta$-sheet a.a. E28 (with R71:NE and R71:NH2 bonds of precisely the same distance) cancels out. More specifically, there are three potential H-bonds with the N30:O (at R71:NH1, R71:NH2, and H96:NE2); Y95 appears to $\mathrm{H}$-bond to $\mathrm{A31}: \mathrm{O}$, and there is also a potential bond between Q70:OE1 and R71:NH1 (Figs. 3A \& D). By contrast, $1 F Y T-V \beta$ displays single $H$-bonds between T97:O and N312:ND2 (peptide), and G98:O and K315:N (peptide). The DRB1, R71:NH2 is involved with $\mathrm{H}$-bonding to $\mathrm{N} 312: \mathrm{O}$, and R71:NH1 has possible H-bonds with N312:OD1 and with Q70:OE1 (Figs. 3B \& E). Since 1FYT and 1J8H only differ via DRB1 alleles, we compared H-bonding for the two (Fig. 3B vs. 3C; 3E vs. 3F). Illustrating the importance of the Swiss computation, in Figure $3 \mathrm{C}$ it was anticipated that the R71K polymorphism would effect three $\mathrm{H}$-bonds-involving $\mathrm{K} 71$ in a manner similar to R71. However, as is shown in Figure 3F, R71K actually shifted peptide contacts to Q70; in-fact, K71 did not show any $\mathrm{H}$-bonds. Importantly, this confirms the change in peptide conformation between the two structures as originally reported [13], and suggests that alloreactivity of $1 \mathrm{FYT} / 1 \mathrm{~J} 8 \mathrm{H}$ involves the single $\mathrm{G} 98: 0$ to $\mathrm{K} 315: \mathrm{N}$ bond in maintaining the $\approx 1740 \AA^{3} d \mathrm{~V}$ of the two $\mathrm{V} \beta$ (Table 1B). Still, the chemistry of each possible bond must be examined to arrive at feasible mechanisms; arguably, this is necessary to understand binding. 

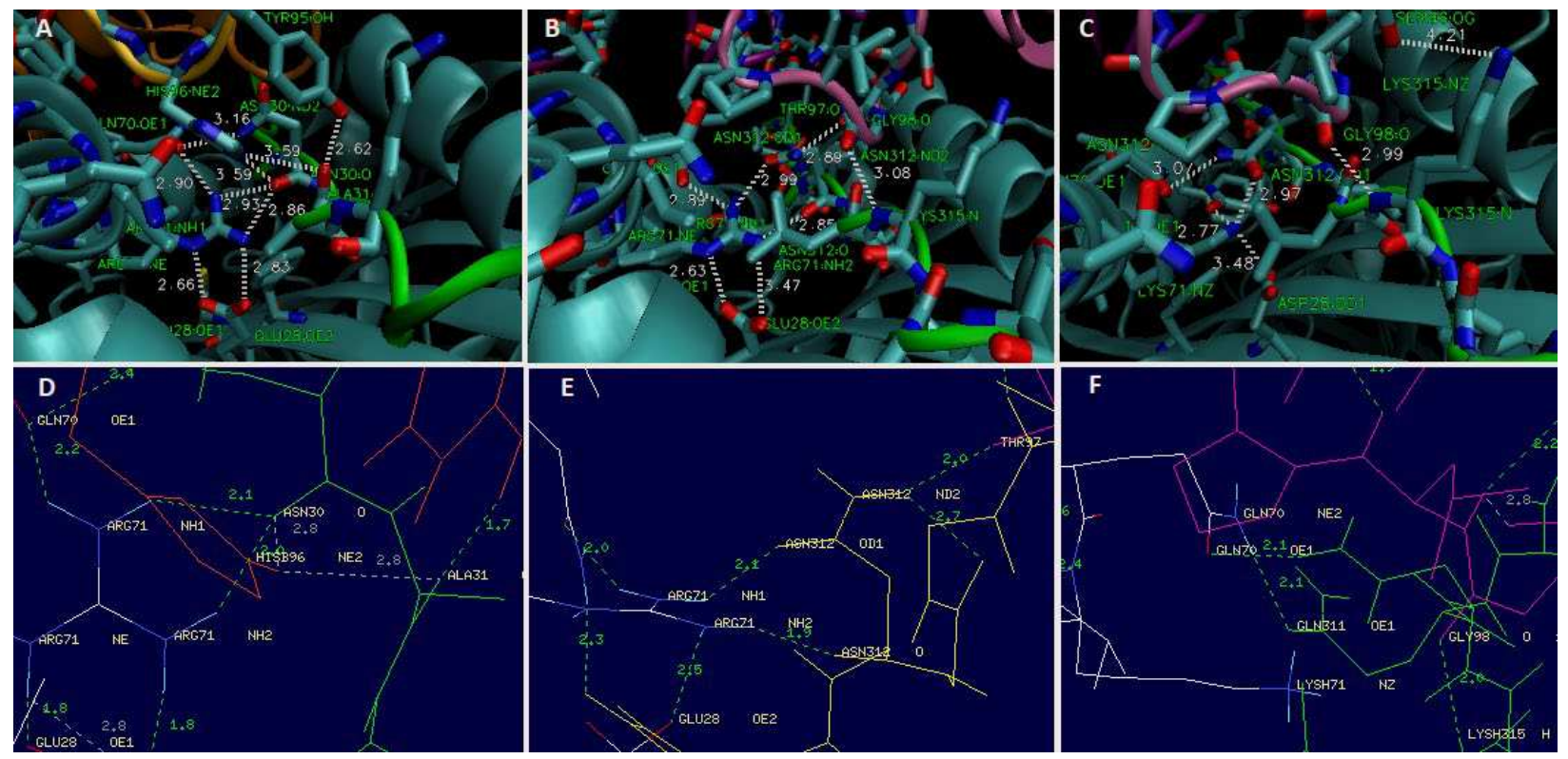

Figure 3 CDR3 $\mathrm{H}$-bonding networks of $2 \mathrm{IAM}-\mathrm{V}_{\boldsymbol{\beta}}(\mathrm{A} \& \mathrm{D}), 1 \mathrm{FYT}-\mathrm{V}_{\boldsymbol{\beta}}$ (B \& E) and $\mathbf{1 J 8 H} \mathbf{8} \boldsymbol{\beta}$ (C \& F). PDB files analysed with VMD (top) and Swiss-Deepview (bottom). In Swiss, hydrogens added and $\mathrm{H}$-bond distances computed; see dotted lines in green. Involved side chains shown by atom in licorice (top) or stick (bottom); The 2IAM CDR3 $\beta$ in gold (A) and red (D); $1 \mathrm{FYT} / 1 \mathrm{~J} 8 \mathrm{H}$ CDR3 $\beta$ in mauve (B, C, E, F); and peptides in green. Figure $(A-C)$ are the original output of the indicated $P D B$ file as analysed in VMD1.9.1. Figure (D-F) are the original $P D B$ outputs as analysed in Swiss-Deepview-v4.1. Best viewed at $125 \%$.

\section{H-Bonding Mechanisms}

Shown in Figure 4A is a suitable reaction mechanism for the 2IAM CDR3 $\beta$ binding to the peptide (triosephosphate isomerse, 15mer)-HLA-DR complex (PDB ref., 15). When considering the probability of hydrogen bonds in a mechanism, all possibilities were drawn using ChemSketch (ACD Labs; www.acdlabs.com/resources) and electron pathways traced using standard evaluations of electron configuration [24, 25]. Thus DRB1 (B1) Q70:OE1 would be in an H-bond with B1R71:NH1 in the absence of the peptide, with the downstream effect of relieving the charge on the B1R71:NH2. Bound peptide has the same effect on R71:NH2, where the N30:O attacks the R71:NH2 (orange arrows). Here, the peptide would indirectly break the H-bond between Q70 and R71 (orange blocks). When the TCR binds, the $\beta$ H96:NE2 charge is preferentially attacked by the N30:O (purple arrows); this reverses the previous bond between the peptide and DRB1 (purple block) and has the downstream effect of re-forming the intraMHC bond between Q70 and R71 (purple blocks on the orange blocks). Note, this is the only obvious mechanism that relieves both the $\mathrm{H} 96$ and R71 charges, and would be favoured over an A31:O attack on H96:NE2 by the neighbouring $\beta Y 95 \mathrm{H}$-bond with A31:O (Fig. 4A). Therefore, just three of the possible $\mathrm{H}$-bonds are predicted; and the overall effect is a charge-relay [28, 32, 33] between the MHC, the peptide, and the TCR.

Physiologically, the charge on R71:NH2 could be initially neutralized via the neighbour Q70:OE1-subsequently replaced during processing by CLIP, and/or at HLA-DM exchange, with 
antigenic peptide (ref. 9). During T-cell conjugation with the antigen presenting cell, the TCR would replace the A30:O to R71:NH2 attack with an A30:O to H96:NE2 attack, which relays the charge back to R71:NH2-subsequently, neutralized again by Q70:OE1. While the hallmark charge-relay network of the 'catalytic triad' within serine proteases stabilizes formation of a covalent acyl-enzyme intermediate [24]; here, a relay is utilized in a similar (albeit, noncovalent) role. Undoubtedly, this would still be a particularly stabilised transition-state, i.e., given that these $\mathrm{H}$-bonds are de-localised across $3 / 5$ components of the structure [24-27].

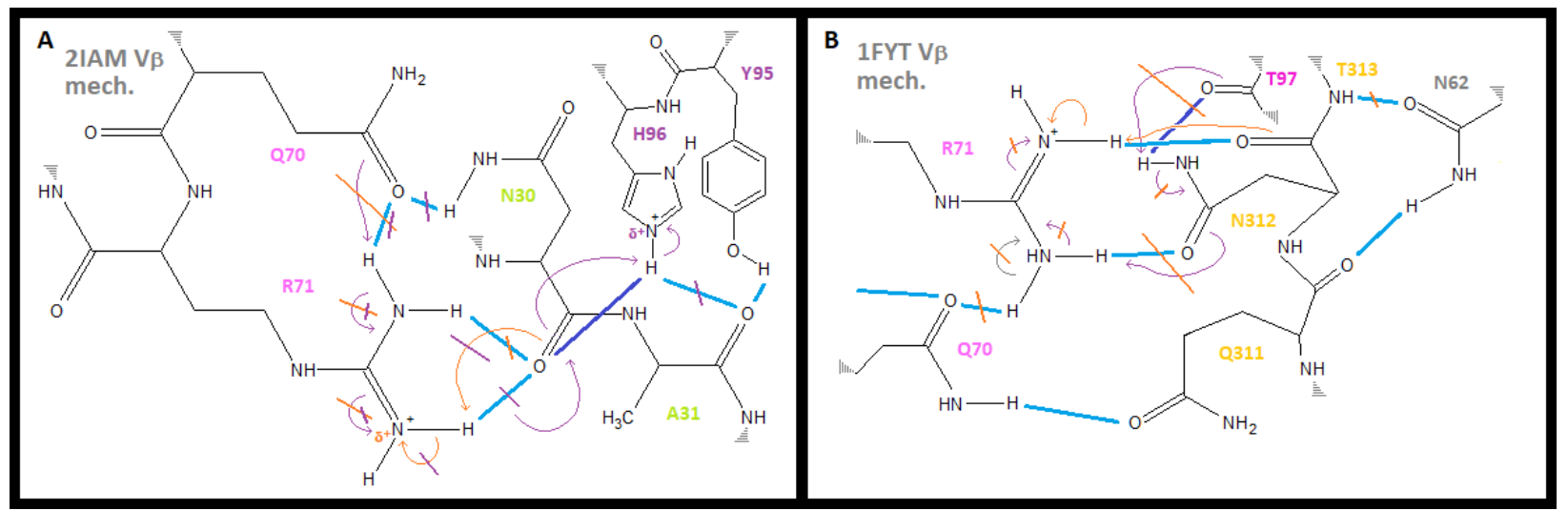

Figure $4 \mathrm{H}$-bonding mechanism for CDR3 $\beta$ binding to peptide and MHC. ChemSketch drawn mechanisms are shown for 2IAM (A) and 1FYT (B) and were based on analyses in VMD and Swiss (Fig. 3). Not necessarily to scale. Electrons shown with arrows (purple, involving the TCR; orange, related to the peptide). Possible H-bonds are shown in blue (darker shade for key TCR bond) and are crossed with the color-coded block line if not probable (see text). Best viewed at $125 \%$.

By contrast, Figure $4 \mathrm{~B}$ shows a suitable mechanism for the analogous CDR3 $\beta$ binding reaction with the influenza HA peptide-HLA-DR complex of 1FYT. The differences are the peptide and TCR, as both 2IAM and 1FYT are DRB1*0104 structures. Similarly the peptide would replace the internal B1Q70:OE1 to B1R71:NH1 with an H-bond directly with the charged R71:NH2 via the peptide N312:0 (orange arrows). Again, the TCR could replace this bond with an attack by the CDR3 $\beta$ (here, T97:O on N312:ND2; purple arrows). However, the downstream effect of such an attack in this case is a partial charge on N312:OD1 and an attack on R71:NH1. Note this would restore the charge on $\mathrm{R} 71: \mathrm{NH} 2$, effectively breaking the peptide-MHC bond. Also, despite what looks like proximity in the ChemSketch diagram, T97 is actually far removed from R71 (Fig. 3E) such that an attack directly on R71:NH2 is not possible. Thus, because of a remaining charge on R71:NH2, the T97:O attack on N312:ND2 (with its downstream effects) would not be favoured (orange blocks). While similarly to 2IAM there is a neighbouring $\mathrm{H}$-bond (in this case to Q311), the net loss of a CDR3 $\beta \mathrm{H}$-bond clearly distinguishes the two mechanisms, and shows that the 1FYT CDR3 $\beta$ does not favour a charge-relay mechanism; i.e., would not have the stabilized CDR3 $\beta$ tether at B1R71-ostensibly, essential chemistry driving restricted $d V$ in 2IAM. Note that the 2IAN-V $\beta$ mechanism appears to be identical to that of 2IAM (structures differ by a single, sufficiently distant, a.a. of the peptide). Next, 3TOE also showed the TCR:peptide:MHC:MHC type of potential network by contacts analysis (Table 2B). Figure 5A shows the a.a. involved in this potential network using Swiss, and the proposed mechanism is shown in Figure 5B. Note that the DRB1-4 allotype of 3TOE has the R71K polymorphism, and 
that the involved peptide a.a. is R9. In terms of distinguishing the mechanism from 2IAM, the question is whether the CDR3 $\beta \mathrm{N} 100 \mathrm{H}$-bond to R9 could exist with a B1:peptide $\mathrm{H}$-bond. In fact, if the $\mathrm{V} \beta$ closest contact $\mathrm{N} 100: \mathrm{O}$ is to attack the positive charge of R9:NH2 (purple arrows), it would have to compete (indirectly) with the H-bond of B1Q93:OE1 to R9:NH1 (orange arrows). Together with the B1Q99:NE2 possible bond to the same N100:O, the B1Q93 bond would be favoured; thus, the 3TOE mechanism is not similar to 2IAM, most notably because CDR3 $\beta$ is not likely to form an $\mathrm{H}$-bond with the peptide. Note that in the related structure, $P D B$ 306F, this conclusion holds as well, where B1D95:OD2 has a possible H-bond to R9:NH2 at 1.6 $\AA$; again, not favouring a CDR3 $\beta$ N100:O bond to the peptide [28].

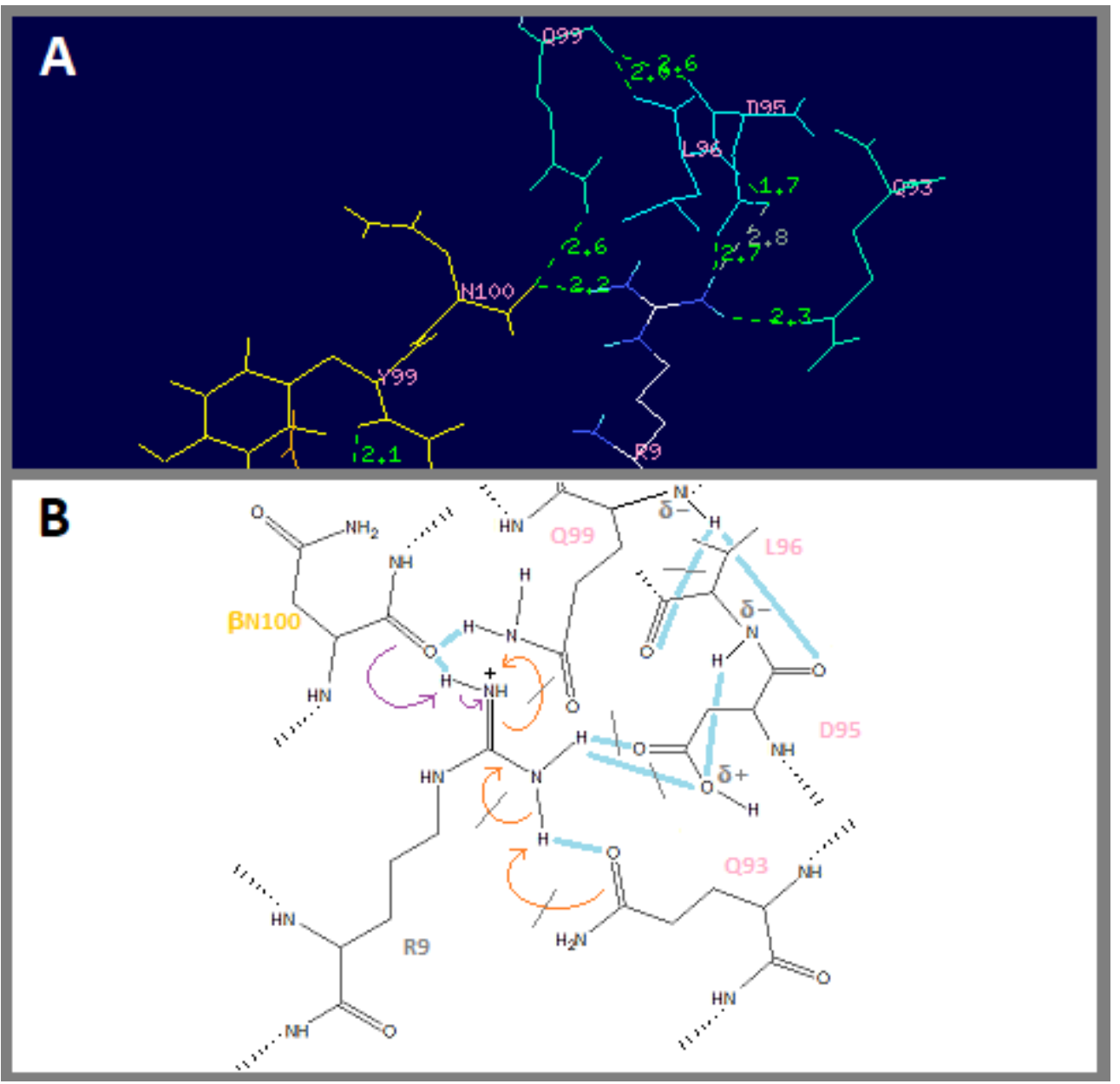

Figure $5 \mathrm{H}$-bonds and H-bonding mechanism for 3T0E CDR3 $\beta$. Swiss was used as before (non-involved a.a. deselected for simplicity) to compute possible H-bonds for 3TOE (A). Mechanism for 3TOE TCR binding via CDR3 $\beta$ to peptide and DRB1 (B) shows possible $\mathrm{H}$-bonds in blue, with improbable bonds blocked with grey lines (ChemSketch). Electron flow shown with purple arrows (TCR), or orange (MHC). CDR3 $\beta$ in yellow (top) or a.a. labelled yellow (bottom); DRB1 in mauve (top) or labelled a.a. in pink (bottom); peptide by atom (top) or a.a. labelled grey (bottom). Figure (A) is the original output of the $P D B$ file as analysed in Swiss-Deepview-v4.1. Figure (B) is a diagram constructed in ChemSketch. Best viewed at $100 \%$.

\section{DQ H-Bonding Mechanisms}


400

401

402

403

404

405

406

407

408

409

410

411

412

413

414

415

416

417

418

419

420

421

422

423

424

425

426

427

428

429

430

431

As shown in Table 1D, we noted the highly-restricted $d V$ in V $\beta$ for two of the TCR:pHLA-DQ structures (4OZH \& 4OZG; $267 \AA^{3}$ and $276 \AA^{3}$, respectively) [PDB ref. 29]. Here, the control structure is $4 \mathrm{OZF}\left(\mathrm{V} \beta d V, 554 \AA^{3}\right)$, where the pMHC is the same; in-fact, these three structures only differ by their CDR3 regions (all 38 IMGT-computed CDR3 junctions shown in Suppl.1.III). Accordingly, $\mathrm{H}$-bonding was analysed with Swiss-Deepview as before (Fig. 6). While very similar, we noted the absence (Fig. 6A \& B) of the DQA1, T61:OG1 role in H-bonding to V $\beta$, R109:NH1 (Fig. 6C). This is accomplished in 4OZH by preferential H-bonding to $\mathrm{V} \beta$ a.a. S110 (Fig. 6A), or sterically in $4 O Z G$ by $V \beta$ a.a. F110 (Fig. 6B). Thus, only in 4OZF is T61:OG1 free to Hbond to the pivotal arginine (CDR3 $\beta$, R109); note, this shift from R109 contacting MHC at a.a. N62 to T61 indirectly disrupts the MHC:MHC component of the charge-relay mechanism (Fig. 7C vs. 7A \& B). Crucially, the chemistry of S110 versus F110 versus A110 in these TCR is ultimately the result of somatic genetic construction of the CDR3 $\beta$ loop [Suppl.1.III; ref. 4].

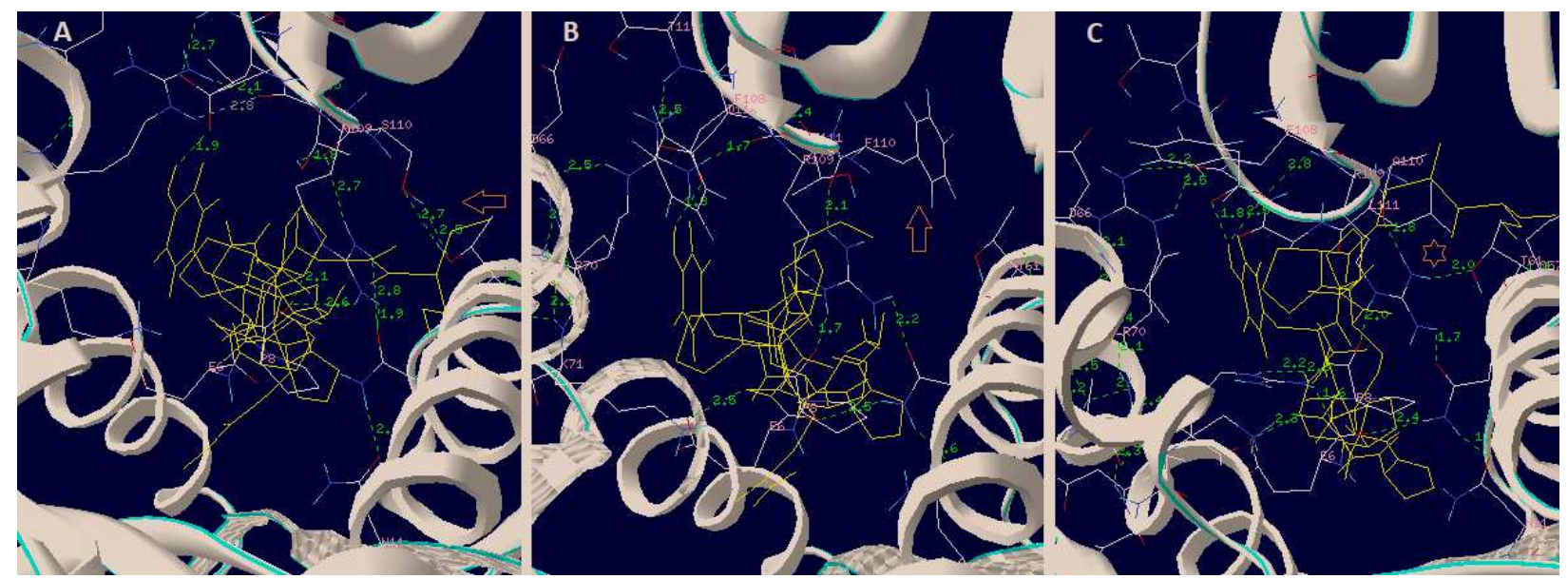

Figure $6 \mathrm{H}$-bonds for three different TCR CDR3 $\beta$, (from germline-identical TCR) on the same pHLA-DQ. (A) 4OZH, showing S110:T61 H-bonds (orange arrow), (B) 4OZG, showing bulky F110 (orange arrow); both of which could preclude (C), the conserved R109 H-bonds to T61 (orange star). Swiss-Deepview analysis as previously; $\mathrm{DQ}$ and CDR3 $\beta$ backbones in white ribbon; peptides in yellow wire; involved side chains in CPK; computed possible $\mathrm{H}$-bonds and distances in green. Figures are the original output of the indicated $P D B$ files as analysed in Swiss-Deepview-v4.1. Best viewed at $150 \%$.

Again, a sequentially mechanism can be envisioned. First, there is internal MHC:MHC Hbonding between N11 and N62 (purple arrows, Fig. 7A \& B). This facilitates the TCR binding to peptide via a P8:O attack on the R109:NE proton (blue arrows), and this leads to neutralizing the charge on R109: $\mathrm{NH} 2$. Uniquely here (purple arrows, Fig. 7C), when the CDR3 $\beta$ is permissible for the T61:OG1 to R109:NH1 H-bond, i.e., the loop a.a., A110-L111-A112-A113 (4OZF), this appears to preclude both N62:OD1 attack on the charged R109:NH2 and the MHC-MHC H-bond (N11:OD1 to N62:ND1); compare in Fig. 7C to 7A \& B). Thus, all three TCR (across DR and DQ) which displayed highly-restricted $d V$, also shared the likelihood of a charge-relay mechanism of CDR3 $\beta$ binding, where an $\mathrm{MHC}$ to $\mathrm{MHC}$ bond is involved. 


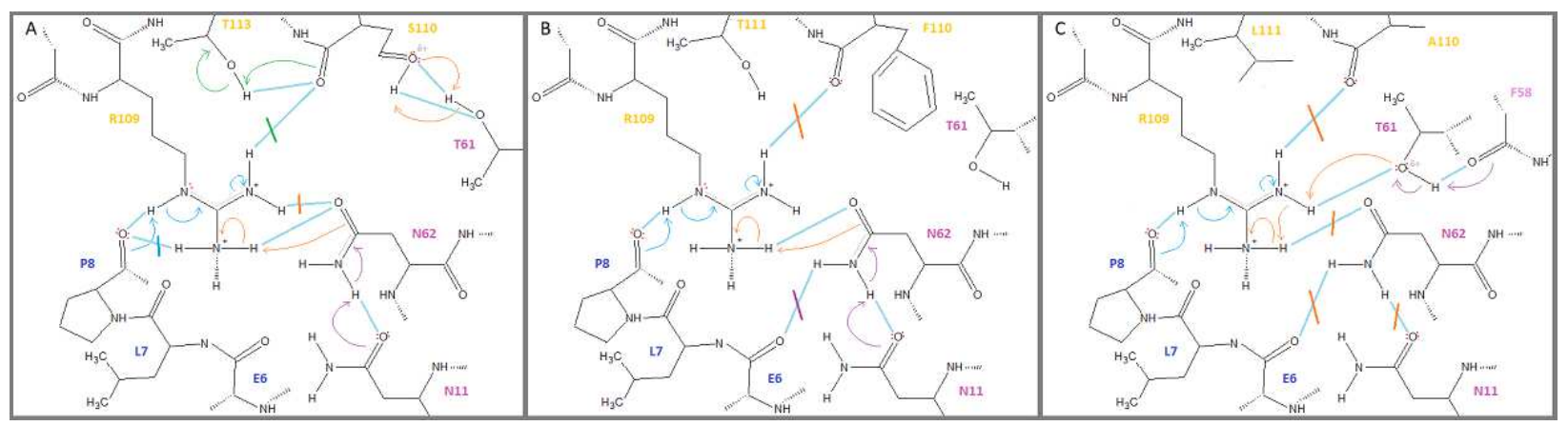

Figure $7 \mathrm{H}$-bond mechanisms for three different CDR3 $\beta$, (from germline-identical TCR) on the same pHLA-DQ. (A) 4OZH, showing S110:T61 H-bonds (orange arrows), (B) 4OZG, showing bulky F110; both of which could preclude (C), the conserved R109 H-bonding to T61, as only observed for the nominal $d \mathrm{~V}$ $\mathrm{V} \beta$ of $4 \mathrm{OZF}$. As previously, possible $\mathrm{H}$-bonds in blue, with improbable bonds blocked with colourmatched lines (ChemSketch). Electron flow shown with blue arrows (peptide:TCR), or orange arrows (MHC:TCR), or purple arrows (MHC:MHC). CDR3 $\beta$ a.a. labels in yellow, peptide a.a. in blue, DRB1 a.a. in mauve. Best viewed at $150 \%$.

\section{Discussion:}

We used spherical coordinates to derive a unique solved triple integral for each of the $38 \mathrm{~V}$ domains (Figs. 1 \& 2; Suppl.1.I). Using this approach the mean volumetric-density of a TCR Vdomain through the putative CDR2 scanning path was $1060 \AA^{3}$. As indicated, $\iiint_{V} d V$ is a 'slice' volume element of a cone with the vertex at the 'across-the-groove' MHC $\alpha$-helix, where maximal CDR2 scanning $(d \theta)$ calculates close to the actual range of $\alpha$-helix distances between the most $\mathrm{N}$-term. and most $\mathrm{C}$-term. CDR2 contacts (Tables 1A-1D). Also, overall geometry consistent with MHC tethering via CDR3 (first formulated for TCR-V $\alpha$ :pHLA-A2; ref. 20) was again, broadly apparent. Most importantly, here a linear relationship was found between $\mathrm{V}$ domain pitch; calculated by:

$$
\varphi_{c}=\mathbf{k} \varphi_{m}=[\sigma \div(\lambda+\sigma)] \omega
$$

and V-domain " $d V$ " calculated by:

$$
\iiint_{V} d V=\int_{0}^{\sin ^{-1}(r / \rho)} \int_{0}^{\pi \cdot r / 4} \int_{0}^{z / \cos \phi} \rho^{2} \sin \phi d \rho d \theta d \phi
$$

This suggests that reduced pitch limits $d \theta$, i.e., the CDR2-scanning function. In retrospect, this might confirm intuition (excluding the broader TCR-CD3 complex, of course) on possibilities for a mechanism involving just the five components. Indeed, $\mathrm{H}$-bonding chemistry which effects relatively simple physics is a hallmark of quite diverse protein machinery [24, 30]. Also, TCR had $k$ values that varied in either direction-indicating that conformation adjustments might moderate flush and open pitch without much increasing or decreasing of the $d V, v i z ., R^{2} \approx 0.900$ (Suppl.1.II). Thus, pitch calculated from a given V-domain's twist-tilt-sway (orientation) might be a kind of "hidden" correlate of TCR-selection; although there is not available crystallography on any thymic (selecting) ligands [31]. For 2IAM, there are data for the uncomplexed TCR, and the CDR3 $\beta$ backbone is displaced $\approx 3.4 \AA$ upon binding $\mathrm{pMHC}$, while $\beta Y 95$ moves $\approx 9.0 \AA$ to form 
508

509

510

the $\mathrm{H}$-bond shown (Fig. $3 \mathrm{~A}$ ); indeed, the key to understanding the TCR may be in this relationship between the "two" induced-fit TCR conformations [32-35]. Suggested here is a conservation/approximation of $d V$ for V-domains in TCR binding the selecting (thymus) and activating (peripheral) pMHC. As shown (Fig. 3E vs. 3F), alloreactivity displays shared $d V$ accomplished by quite different binding chemistry. Alternatively/historically, intrinsic TCR affinity (i.e., equilibrium and rate binding constants specified by the $\alpha / \beta$ TCR protein) has been used to explain both thymic TCR selection and peripheral TCR recognition [4, 36, 37]. Clearly TCR distinguish rare target (agonist) PMHC from thousands of nonagonist PMHC on the APC surface; and this is not mutually exclusive of whether or not a physical force (TCR loading) is an obligate component of TCR function [8, 37]. In these and other regards, there is a stereochemical alternative to an affinity-limited binding reaction [25-27].

Briefly, we assume TCR:pMHC reactions involve a high(er) energy "scanning" conformerbecause, scanning (leading to a suitable CDR2:MHC interface) has the effect of lowering the transition-state free energy, $\Delta \Delta G^{\neq}$(i.e., Curtin-Hammett control) $[25,27,38]$. For example, in TCR like E8 (of 2IAM/2IAN) very little CDR2 $\beta$ scanning is apparently needed, due to the effect of the proposed charge-relay stabilised CDR3 $\beta$ :pHLA-DR transition-state. Thus, $d V$ is a universal consequence of CDR3 chemistry; but usually the reaction requires more CDR2-scanning (i.e., usually V-domains have larger $d V$ values; Tables 1A-1D). Recently, ensemble refinement of crystal data with MD simulations has suggested conformational diversity in the microsecond range, but the conformational changes implicated here-in would be on the millisecond to minutes scale; in this regard, NMR of membrane-bound receptors offers promise [39], and may elucidate the potential of internal water(s) in these H-bonding networks [32]. Finally, spherical coordinates have been used by others to calculate TCR center-of-mass variation in TCR:pMHC complexes [40]. These investigators did not consider $V_{\alpha}$ and $V \beta$ independently, although Hoffmann et al., had shown the angle between $\mathrm{V} \alpha$ and $\mathrm{V} \beta$ characterized different TCR in PMHC complexes; allowing these researchers to group a panel of TCR into six different clusters linked to clonotype [41]. While there are myriad downstream implications, taken together, these data support that V-domain rotation and germline to germline contacts between TCR and PMHC both depend upon CDR3 $\mathrm{H}$-bonding with highly-conserved $\mathrm{MHC} \alpha$-helix motifs. Thus, unlike TCR-affinity, V-domain dynamics are clonotypic. In this regard, similar to the class-I, R65-motif $[20,42]$, the class-II motifs presented here are found in Galago sp. (NCBI Acc. No. AAA96291) as well as in both tarsiers and lemurs; suggesting conservation for at least 63 million years [43, 44].

\section{Methods:}

Availability of data and materials: $P D B$ files are public and available at www.ncbi.nlm.nih.gov; all analytics data are either in the paper, or supplementary materials; Competing interests: Dr. Murray and Xenolaüs Genetics LLC declare no competing interests regarding the research, or its publication; Author Contributions: J.S.M. did the research and wrote the manuscript.

\section{PDB Analysis}


VMD-1.9.1 software (www.ks.uiuc.edu) was used for PDB files downloaded via NCBI (www.ncbi.nlm.nih.gov) from the RSCB-PDB (www.rscb.org); views normalized with the VMD $x y z$-axis tool; alpha carbon $(C \alpha)$ main-chains in new cartoon; all alleles named per $N C B I$ annotation. Euler's methods (www.mathword.wolfram.com/EulerAngles.html) were the basis for the specific angle analyses, as previously reported. Briefly, three angles corresponding to the twist, tilt, and sway of each domain over the pMHC were measured from fixed $\mathrm{C} \alpha$ through the 19 structures: (i) in-plane to the MHC-groove $\{$ twist $=\omega\}$, with (ii) displacements perpendicular to the groove $\{t i l t=\lambda\}$, including (iii) side-to-side variation $\{$ sway $=\sigma\}$. The a.a. positions used as coordinates for angular measures across structures were fixed; see previous. The incline of a V-domain, $\left\{\right.$ pitch $\left.=\varphi_{\mathrm{m}}\right\}$, was approximated (calculated) by the equation: $\varphi_{\mathrm{c}}=[\sigma$ $\div(\lambda+\sigma)] \omega$ (see also Results, Tables 1A-1D). Pitch was also measured by using the closest determined CDR2 contact $\mathrm{C} \alpha$ for an angle across-the-groove to the closest CDR3 contact with an $\alpha$-helix side-chain (vertex), then back to said CDR2 closest contact within the opposite $\alpha$ helix $(\approx 2$-fold symmetry); angular value in degrees via the VMD angle-label tool. Triple integrals for all $38 \mathrm{~V}$-domains based on the fixed geometry were solved as described in Results (Suppl.1.I). Linear regression analysis by MS-Excel (Suppl.1.II); and statistics by paired twotailed Student's t-test (www.insilico-net/tools/statistics/ttest).

\section{Contacts Analysis}

All measures were performed with Swiss or VMD-1.9.1 as is specified in Results. Closest contacts in angstroms $(\AA)$ were determined by examining appropriate coordinates between structures (computed in Swiss, or measured/computed in VMD). Individual atomic contacts are named per software annotation. DRA chain contacts are abbrv. "A1" to avoid confusion with single-letter a.a. code for alanine $(A)$; potential $\mathrm{H}$-bond networks are colour-coded as is described in table captions and the text.

\section{CDR3 Joint Analysis}

Nucleotide sequences for all CDR3 of TCR were specified from PDB files (Suppl.1.III). TCR a.a. sequences were reverse translated using the SMS tool at www.bioinformatics.org. These were then imported into IMGT algorithms for joint analysis (www.imgt.org). Amino acid sequences of resulting CDR3 joints were determined by the IMGT algorithm; consensus IMGT numbering.

\section{H-Bonding Mechanisms}

Hydrogen $(\mathrm{H})$ bonds were estimated either with $V M D$, or (more accurately) with Swiss. In Swiss, $\mathrm{H}$-bonds are computed after computing hydrogens to the structures. H-bond distances were used as a factor in determining suitable organic reaction mechanisms, where relevant side-chains were reproduced with Chemsketch (www.acdlabs.com/resources). Standard evaluations of electron configuration per relevant atoms were used to predict electron flows [25]. 
554

555

556

557

558

559

560

561

562

563

564

565

566

567

568

569

570

571

572

573

574

575

576

577

578

579

580

581

582

583

584

585

586

587

588

589

590

591

592

593

594

595

\section{Acknowledgements:}

Crystallographers, molecular biologists and computer scientists providing public availability of coordinates, sequences and software made this work possible. Thank you.

\section{Supplementary details:}

[Suppl.1.pdf] I. Triple Integral Solutions for all V-domains

II. Linear Regression Analysis of Calculated Pitch versus $d V$ (all V-domains)

III. IMTG CDR3 Joint Analysis (all V-domains)

\section{References:}

1. Allison, J. P., Mclntyre, B. W. \& Bloch, D. Tumor-specific antigen of murine T-lymphoma defined with monoclonal antibody. J. Immunol. 129, 2293-300 (1982).

2. Tonegawa, S. Somatic generation of antibody diversity. Nature. 302, 575-81, doi: 10.1038/302575a0 (1983).

3. Agrawal. A., Eastman, Q. M. \& Schatz D. G. Transposition mediated by RAG1 and RAG2 and its implications for the evolution of the immune system. Nature. 394, 744-51, doi: 10.1038/29457 (1998).

4. Janeway, C. A., Jr. Immunobiology. 5th ed. (Garland, New York, 2001).

5. Zinkernagel, R. M. \& Doherty, P. C. Restriction of in vitro T cell-mediated cytotoxicity in lymphocytic choriomeningitis within a syngeneic or semiallogeneic system. Nature. 248, 701-2, doi: 10.1038/248701a0 (1974).

6. Galvez, J., Galvez, J. J. \& Garcia-Penarrubia, P. Is TCR/pMHC Affinity a Good Estimate of the T-cell Response? An Answer Based on Predictions From 12 Phenotypic Models. Front. Immunol. 10, 349, doi: 10.3389/fimmu.2019.00349 (2019).

7. Gee, M. H., Sibener, L. V., Birnbaum, M. E., Jude, K. M., Yang, X., Fernandes, R. A., et al. Stress-testing the relationship between $\mathrm{T}$ cell receptor/peptide-MHC affinity and crossreactivity using peptide velcro. Proc. Natl. Acad. Sci. USA. 115, E7369-78, doi: 10.1073/pnas.1802746115 (2018).

8. Hwang, W., Mallis, R. J., Lang, M. J. \& Reinherz E. L. The $\alpha \beta T C R$ mechanosensor exploits dynamic ectodomain allostery to optimize its ligand recognition site. Proc. Natl. Acad. Sci. USA. 117, 21336-45, doi: 10.1073/pnas.2005899117 (2020). 
9. Wieczorek, M., Abualrous, E. T., Sticht, J., Álvaro-Benito, M., Stolzenberg, S., Noé, F. \& Freund, C. Major Histocompatibility Complex (MHC) Class I and MHC Class II Proteins: Conformational Plasticity in Antigen Presentation. Front. Immunol. 8, 292, doi: 10.3389/fimmu.2017.00292 (2017).

10. Nisonoff, A., Hopper, J. E. \& Spring, S. B. The Antibody Molecule. 1st ed. (Academic Press, New York, 1975).

11. Brown, J. H., Jardetzky, T., Saper, M. A., Samraoui, B., Bjorkman, P. J. \& Wiley, D. C. A hypothetical model of the foreign antigen binding site of class II histocompatibility molecules. Nature. 332, 845-50, doi: 10.1038/332845a0 (1988).

12. Davis, M. M. \& Bjorkman, P. J. T-cell antigen receptor genes and T-cell recognition. Nature. 334, 395-402, doi: 10.1038/334395a0 (1988).

13. Hennecke, J. \& Wiley, D. C. Structure of a complex of the human alpha/beta T cell receptor (TCR) HA1.7, influenza hemagglutinin peptide, and major histocompatibility complex class II molecule, HLA-DR4 (DRA*0101 and DRB1*0401): insight into TCR cross-restriction and alloreactivity. J. Exp. Med. 195, 571-81, doi: 10.1084/jem.20011194 (2001).

14. Li, Y., Huang, Y., Lue, J., Quandt, J. A., Martin, R. \& Mariuzza, R. A. Structure of a human autoimmune TCR bound to a myelin basic protein self-peptide and a multiple sclerosisassociated MHC class II molecule. EMBO J. 24, 2968-79, doi: 10.1038/sj.emboj.7600771 (2005).

15. Deng, L., Langley, R. J., Brown, P. H., Xu, G., Teng, L., Wang, Q., et al. Structural basis for the recognition of mutant self by a tumor-specific, $\mathrm{MHC}$ class II-restricted T cell receptor. Nat. Immunol. 8, 398-408, doi: 10.1038/ni1447 (2007).

16. Yin, L., Crawford, F., Marrack, P., Kappler, J. W. \& Dai, S. T-cell receptor (TCR) interaction with peptides that mimic nickel offers insight into nickel contact allergy. Proc. Natl. Acad. Sci. USA. 109, 18517-22, doi: 10.1073/pnas.1215928109 (2012).

17. Yin, Y., Wang, X. X. \& Mariuzza, R. A. Crystal structure of a complete ternary complex of Tcell receptor, peptide-MHC, and CD4. Proc. Natl. Acad. Sci. USA. 109, 5405-10, doi: 10.1073/pnas.1118801109 (2012).

18. Sharon, E., Sibener, L. V., Battle, A., Fraser, H. B., Garcia, K. C. \& Pritchard, J. K. Genetic variation in $\mathrm{MHC}$ proteins is associated with $\mathrm{T}$ cell receptor expression biases. Nat. Genet. 48, 995-1002, doi: 10.1038/ng.3625 (2016).

19. Gunnarsen, K. S., Hoydahl, L. S., Risnes, L. F., Dahal-Koirala, S., Neumann, R. S., Bergseng, E., et al. A TCR $\alpha$ frame-work centered codon shapes a biased T cell repertoire through direct MHC and CDR3 $\beta$ interactions. JCI Insight. 2, 95193, doi:10.1172jci.insight.95193 (2017). 
640

641

642

643

644

645

646

647

648

649

650

651

652

653

654

655

656

657

658

659

660

661

662

663

664

665

666

667

668

669

670

671

672

673

674

675

676

677

678

679

680 681

20. Murray, J. S. An old Twist in HLA-A: CDR3 $\alpha$ Hook up at an R65-joint. Front. Immunol. 6, 268, doi: 10.3389/fimmu.2015.00268 (2015).

21. Burrows, S. R., Chen, Z., Archbold, J. K., Tynan, F. E., Beddoe, T., Kjer-Nielsen, L., et al. Hard wiring of $\mathrm{T}$ cell receptor specificity for the major histocompatibility complex is underpinned by TCR adaptability. Proc. Natl. Acad. Sci. USA. 107, 10608-13, doi: 10.1073/pnas.1004926107 (2010).

22. Stewart, J. Calculus. 6th ed. (Brooks/Cole Thomson, Belmont, CA, 2007).

23. Das, B. B., Park, S. H. \& Opella, S. J. Membrane protein structure from rotational diffusion. Biochim. Biophys. Acta. 1848, 229-45, doi: 10.1016/j.bbamem.2014.04.002 (2015).

24. Berg, J. M., Tymoczko, J. L. \& Stryer, L. Biochemistry. 5th ed. (W. H. Freeman, New York, 2002).

25. Carey, F. A. \& Sundberg, R. J. Advanced Organic Chemistry, pt. A. Structure and Mechanism. 2nd ed. (Plenum Press, New York, 1984).

26. Chen, J. \& Thirumalai, D. Interface residues that drive allosteric transitions also control the assembly of I-lactate dehydrogenase. J. Phys. Chem. B. 122, 11195-205, doi: 10.1021/acs.jpcb.8b06430 (2018).

27. Hernández, G., Anderson, J. S. \& LeMaster, D. M. Assessing the native state conformational distribution of ubiquitin by peptide acidity. Biophys. Chem. 153, 70-82, doi: 10.1016/j.bpc.2010.10.006 (2010).

28. Yin, Y., Li, Y., Kerzic, M. C., Martin, R. \& Mariuzza, R. A. Structure of a TCR with high affinity for self-antigen reveals basis for escape from negative selection. EMBO J. 30, 1137-48, doi: 10.1038/emboj.2011.21 (2011).

29. Petersen, J., Montserrat, V., Mujico, J. R., Loh, K. L., Beringer, D. X., van Lummel, M., et al. T-cell receptor recognition of HLA-DQ2-gliadin complexes associated with celiac disease. Nat. Struct. Mol. Biol. 21, 480-8, doi: 10.1038/nsmb.2817 (2014).

30. Brunori, M. The Bohr effect before Perutz. Biochem. Mol. Biol. Educ. 40, 297-9, doi: 10.1002/bmb.20629 (2012).

31. Zerrahn, J., Held, W. \& Raulet, D. H. The MHC reactivity of the T cell repertoire prior to positive and negative selection. Cell. 88, 627-36, doi: 10.1016/s0092-8674(00)81905-4 (1997). 
32. Pusuluk, O., Farrow, T., Cemsinan, D., Burnett, K. \& Vedral, V. Proton tunneling in hydrogen bonds and its implications in an induced-fit model of enzyme catalysis. Proc. R. Soc. A. 474, 20180037, dx.doi.org/10.1098/rspa.2018.0037 (2018).

33. Rudolph, M. G. \& Wilson, I. A. The specificity of TCR/pMHC interaction. Curr. Opin. Immunol. 14, 52-65, doi: 10.1016/s0952-7915(01)00298-9 (2002).

34. Deng, L., Langley, R. J., Wang, Q., Topalian, S. L. \& Mariuzza, R. A. Structural insights into the editing of germ-line-encoded interactions between T-cell receptor and MHC class II by $\mathrm{V} \alpha$ CDR3. Proc. Natl. Acad. Sci. USA. 109, 14960-5, doi: 10.1073/pnas.1207186109 (2012).

35. Buckle, A. M. \& Borg, N. A. Integrating Experiment and Theory to Understand TCR-pMHC Dynamics. Front. Immunol. 9, 2898, doi: 10.3389/fimmu.2018.02898 (2018).

36. Faro, J., Castro, M. \& Molina-París, C. A unifying mathematical framework for experimental TCR-pMHC kinetic constants. Sci. Rep. 7, 46741, doi: 10.1038/srep46741 (2017).

37. Limozin, L., Bridge, M., Bongrand, P., Dushek, O., van der Merwe, P. A. \& Robert, P. TCRpMHC kinetics under force in a cell-free system show no intrinsic catch bond, but a minimal encounter duration before binding. Proc. Natl. Acad. Sci. USA. 116, 16943-48, doi: 10.1073/pnas.1902141116 (2019).

38. Lodola, A., Mor, M., Zurek, J., Tarzia, G., Piomelli, D., Harvey, J. N. \& Mulholland, A. J. Conformational effects in enzyme catalysis: reaction via high energy conformation in fatty acid amide hydrolase. Biophys J. 92, L20-2, doi: 10.1529/biophyj.106.098434 (2007).

39. Mallis, R. J., Brazin, K. N., Duke-Cohan, J. S., Hwang, W., Wang, J. H., Wagner, G., et al. NMR: an essential structural tool for integrative studies of T cell development, pMHC ligand recognition and TCR mechanobiology. J. Biomol. NMR. 73, 319-32, doi: 10.1007/s10858-01900234-8 (2019).

40. Singh, N. K., Abualrous, E. T., Ayres, C. M., Noé, F., Gowthaman, R., Pierce, B. G., et al. Geometrical characterization of $\mathrm{T}$ cell receptor binding modes reveals class-specific binding to maximize access to antigen. Proteins. 88, 503-13, doi: 10.1002/prot.25829 (2020).

41. Hoffmann, T., Krackhardt, A. M. \& Antes, I. Quantitative Analysis of the Association Angle between T-cell Receptor $\mathrm{V} \alpha / \mathrm{V} \beta$ Domains Reveals Important Features for Epitope Recognition. PLoS Comput. Biol. e1004244, doi: 10.1371/journal.pcbi.1004244 (2015).

42. Murray, J. S. Transposon-mediated death of an ancestral A-23-like allele: evolution of TCRpositioning motifs in the HLA-A lineage. Immunogenetics. 67, 473-6, doi: 10.1007/s00251-0150852-3 (2015). 
725 43. Steiper, M. E. \& Seiffert, E. R. Evidence for a convergent slowdown in primate molecular rates and its implications for the timing of early primate evolution. Proc. Natl. Acad. Sci. USA. 109, 6006-11, doi: 10.1073/pnas.1119506109 (2012).

729 44. Kasahara, M., Vazquez, M., Sato, K., McKinney, E. C. \& Flajnik, M. F. Evolution of the major 730 histocompatibility complex: class II A cDNA clones from the cartilaginous fish. Proc. Natl. Acad. 731 Sci. USA. 89, 6688-92, doi: 10.1073/pnas.89.15.6688 (1992). 


\section{Figures}
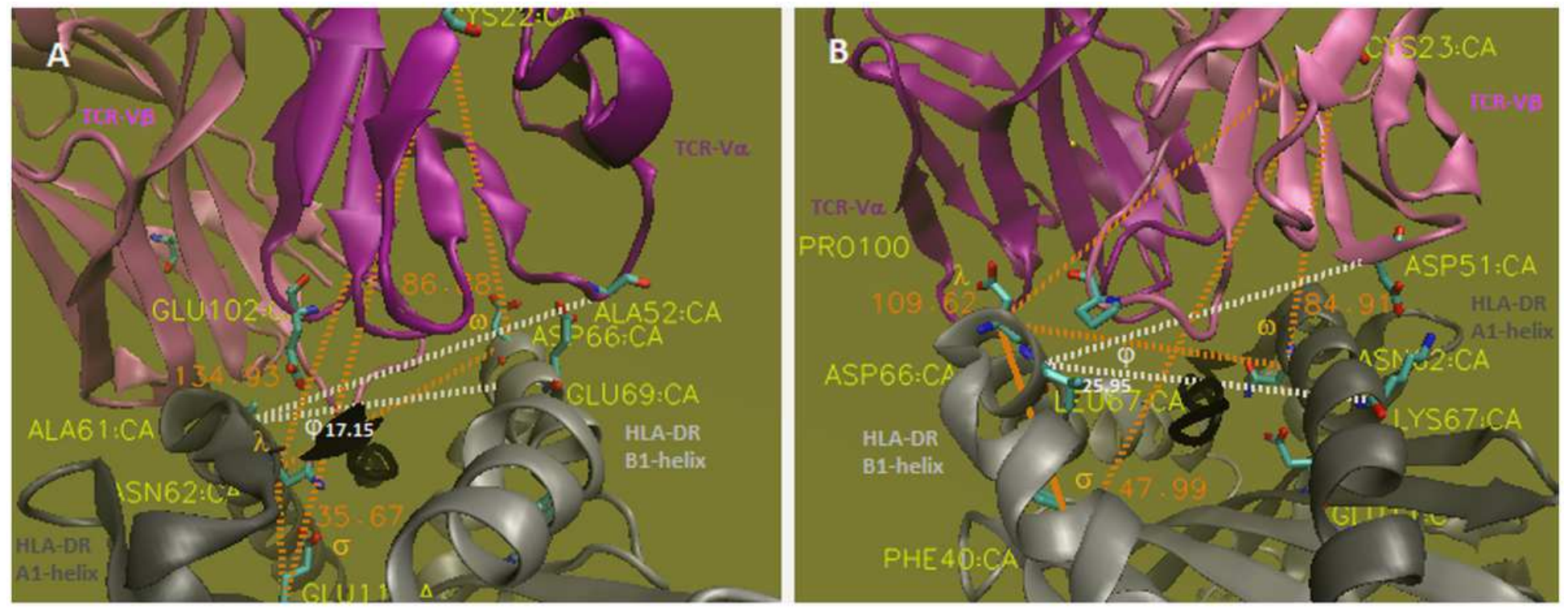

\section{Figure 1}

The twist/tilt/sway of TCR-Va and $-\mathrm{V} \beta$ relative to $\mathrm{pMHC}-\mathrm{II}$ (A \& B, respectively). Please see manuscript .pdf for full caption.

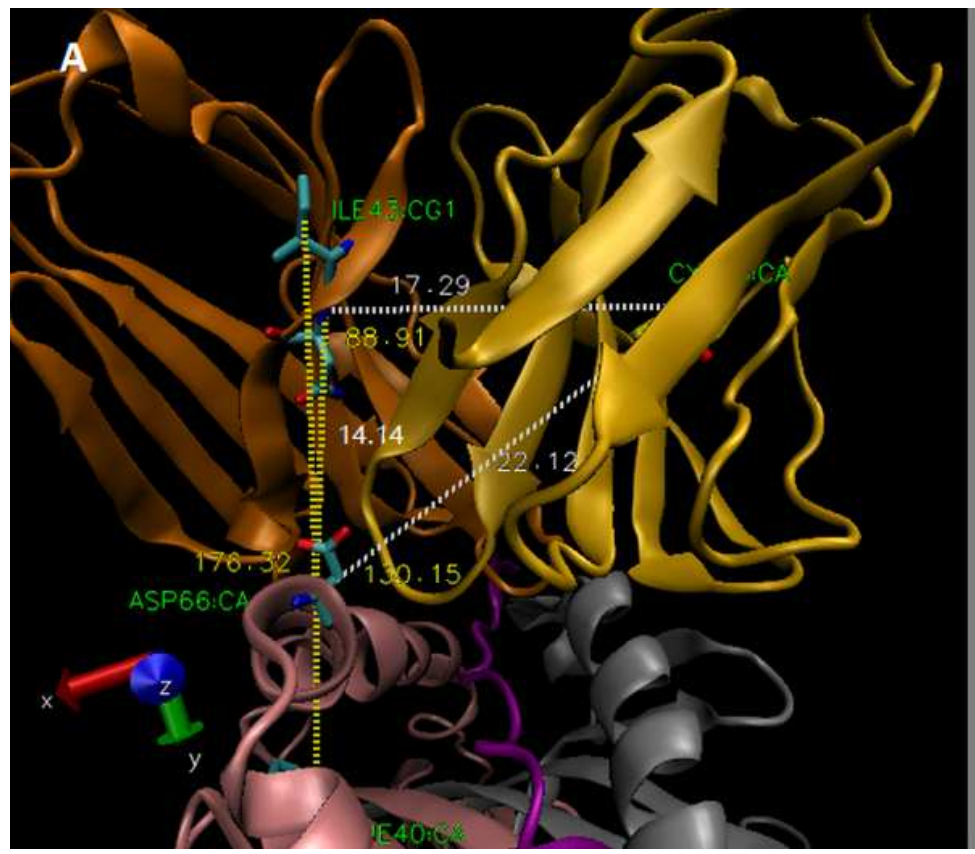

B

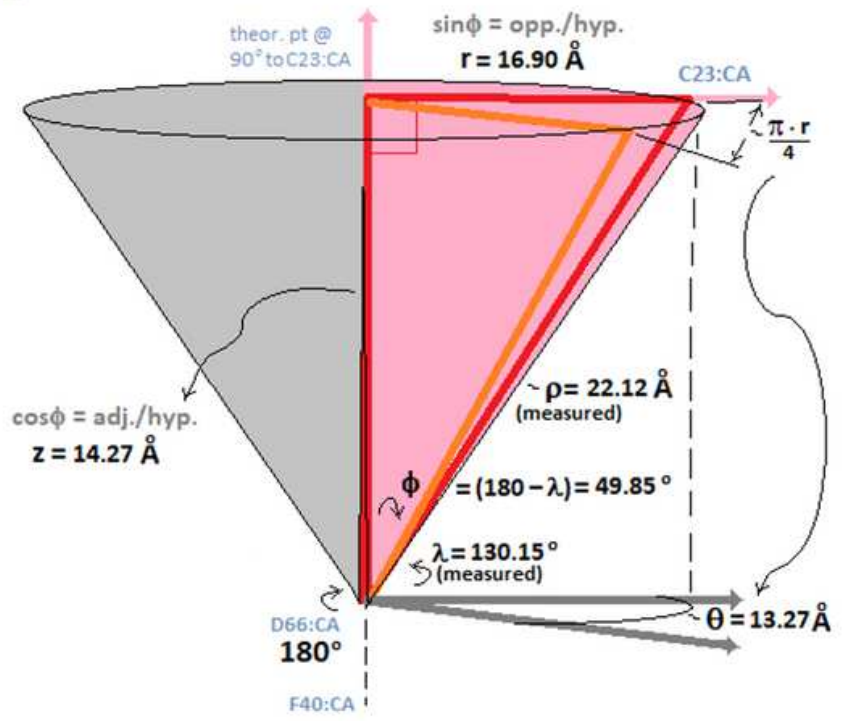

\section{Figure 2}

Derivation of the V-domain rotational volumetric-density equation. Please see manuscript .pdf for full caption. 

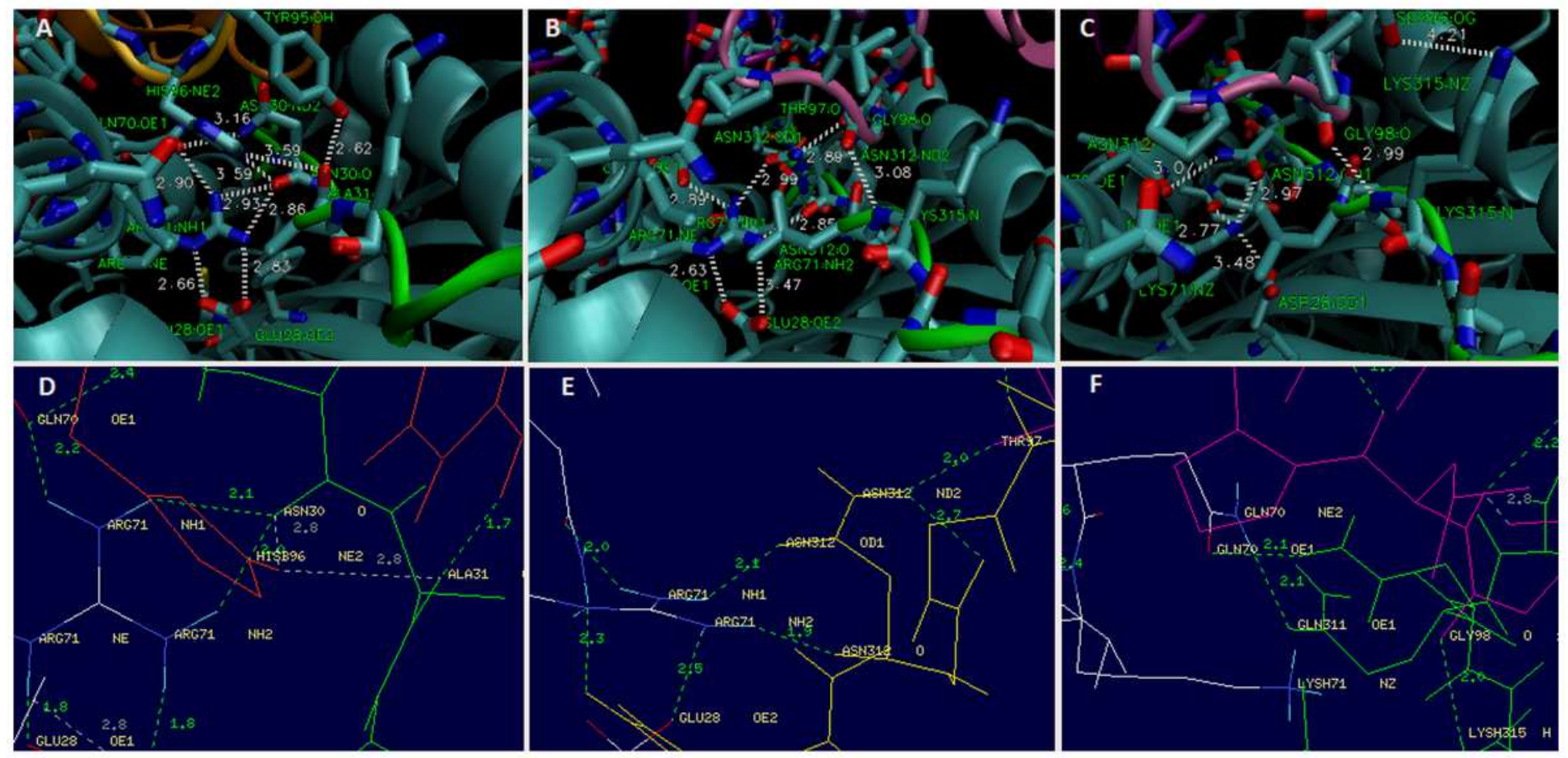

Figure 3

CDR3 H-bonding networks of 2IAM-V $\beta(A \& D), 1 F Y T-V \beta(B \& E)$ and $1 \mathrm{~J} 8 \mathrm{H}-\mathrm{V} \beta$ (C \& F). Please see manuscript .pdf for full caption.

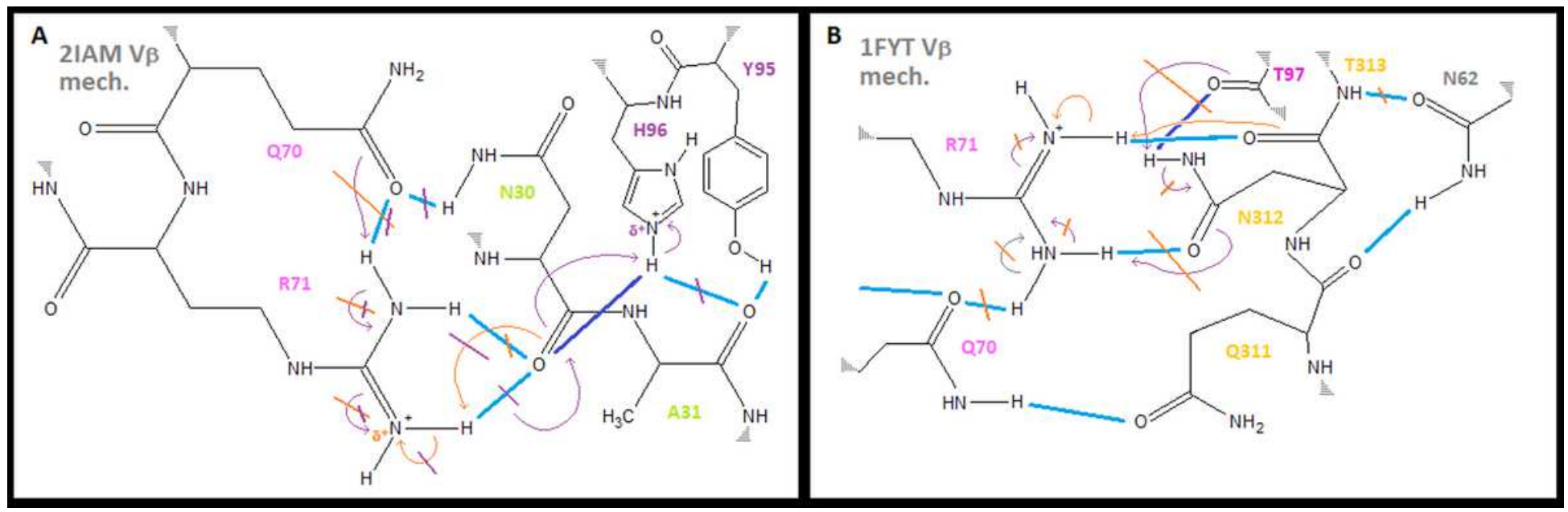

Figure 4

H-bonding mechanism for CDR3b $\beta$ binding to peptide and MHC. Please see manuscript .pdf for full caption. 


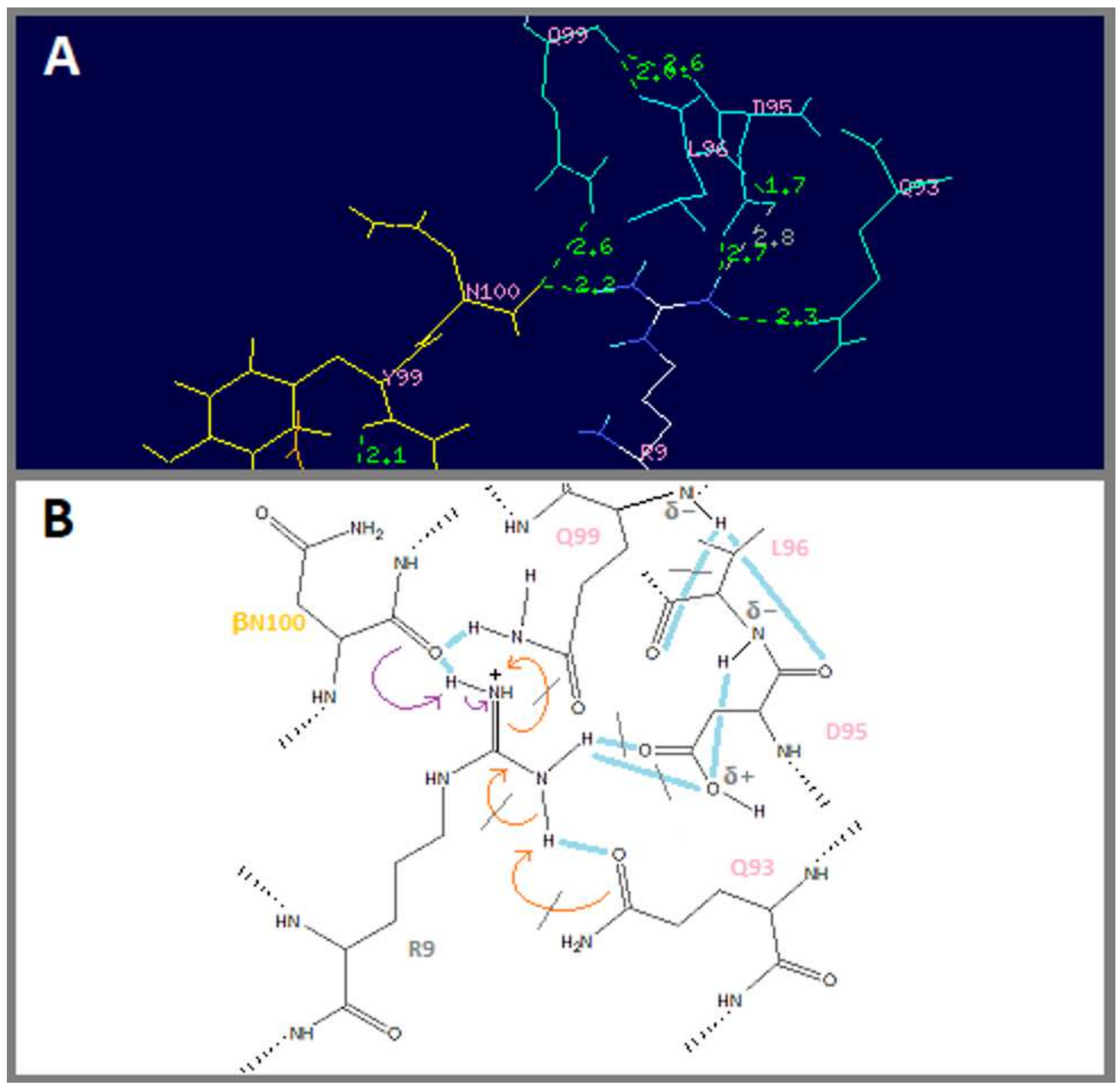

Figure 5

$\mathrm{H}$-bonds and $\mathrm{H}$-bonding mechanism for 3T0E CDR3 $\beta$. Please see manuscript .pdf for full caption. 

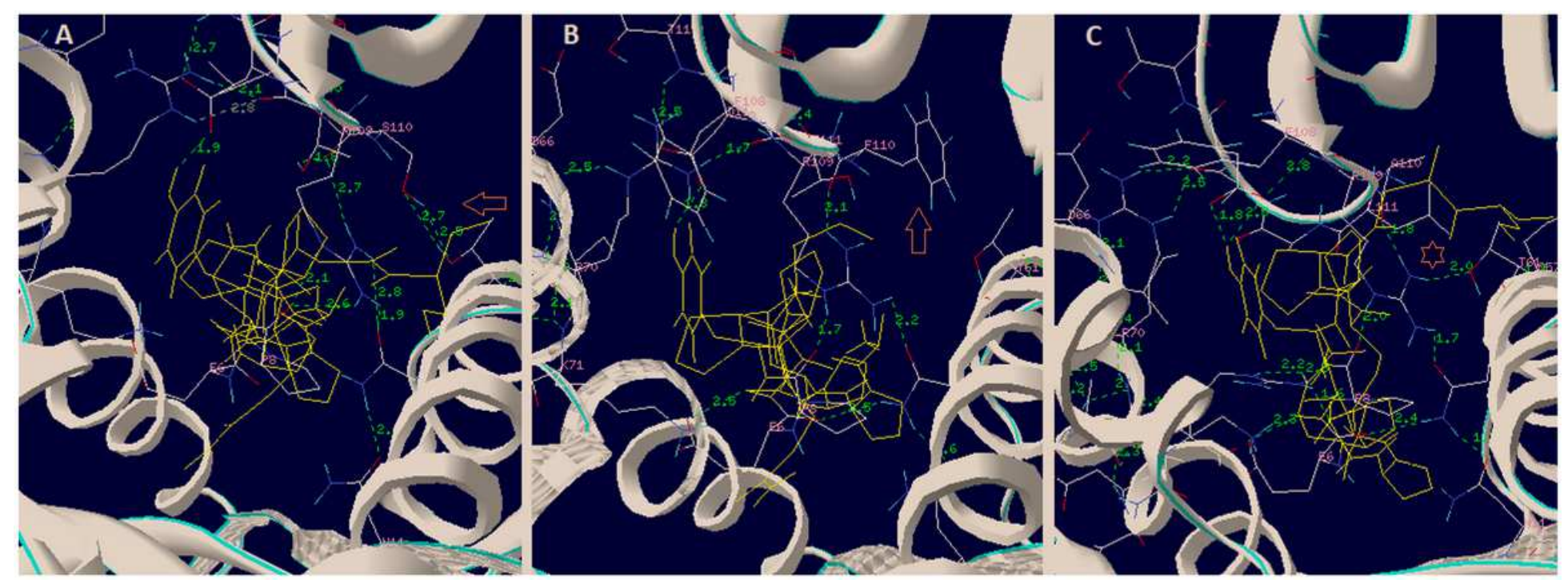

Figure 6

H-bonds for three different TCR CDR3 $\beta$, (from germline-identical TCR) on the same pHLA-DQ. Please see manuscript .pdf for full caption.

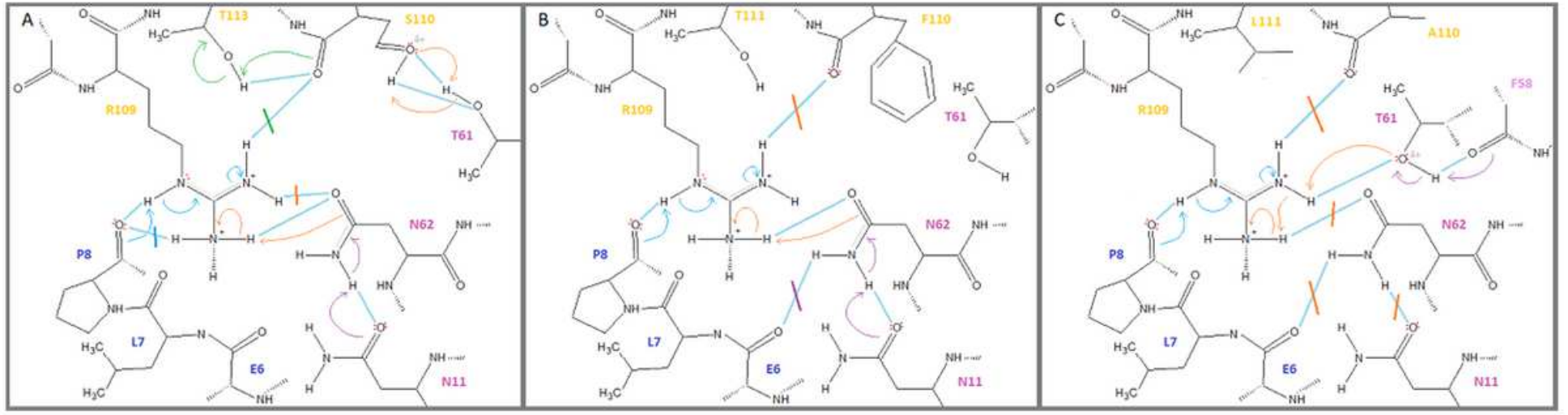

Figure 7

H-bond mechanisms for three different CDR3 3 , (from germline-identical TCR) on the same pHLA-DQ. Please see manuscript .pdf for full caption.

\section{Supplementary Files}

This is a list of supplementary files associated with this preprint. Click to download.

- Suppl.1.pdf 